\title{
Prediction of time of liquefaction using kinetic and strain energy
}

\author{
M.D.L. Millen ${ }^{\mathrm{a}, *}$, S. Rios ${ }^{\mathrm{b}}$, J. Quintero $^{\mathrm{b}}$, A. Viana da Fonseca ${ }^{\mathrm{b}}$ \\ ${ }^{a}$ Department of Civil and Natural Resources Engineering, Faculty of Engineering, University of Canterbury, 20 Kirkwood Avenue, Christchurch, 8041, New Zealand \\ ${ }^{\mathrm{b}} \mathrm{S} / \mathrm{n}$, CONSTRUCT-GEO, Faculty of Engineering, Univeristy of Porto, R. Dr. Roberto Frias, 4200-465, Porto, Portugal
}

\section{A B S T R A C T}

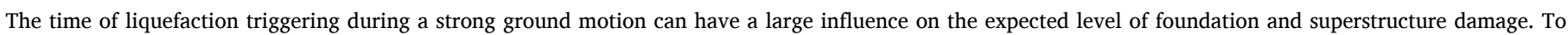

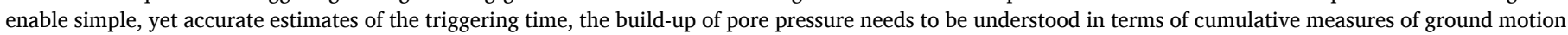

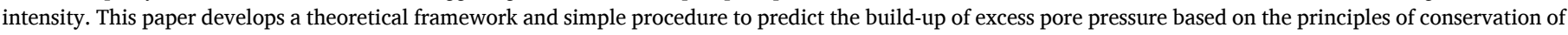

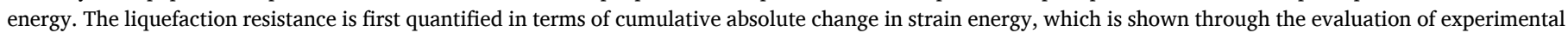

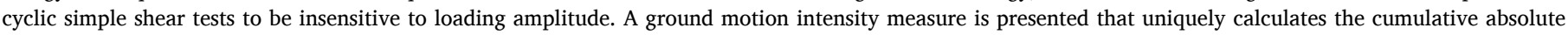

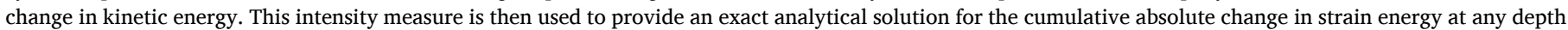

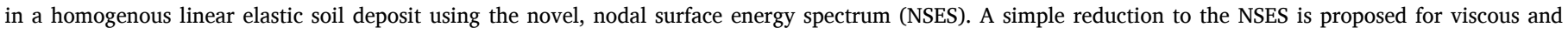

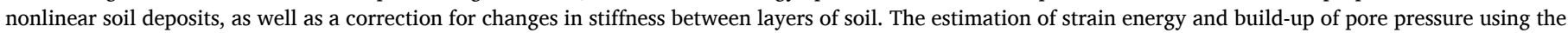

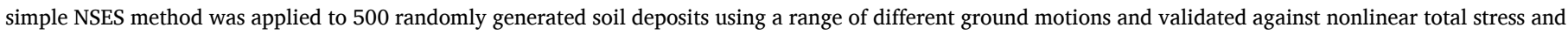

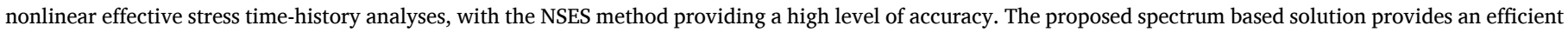
and physically consistent procedure for the prediction of excess pore pressure build-up.

\section{Introduction}

The generation of excess pore water pressure and liquefaction can dramatically change the dynamic response of a soil deposit and interacting structures. Thus the time at which liquefaction occurs, may have a significant influence on the performance of a structure during a seismic event.

Recent work by Wotherspoon et al. [1]; showed that the expected level of surface shaking and seismic energy entering the building is strongly conditioned on the time of liquefaction. In fact Kramer et al. [2], demonstrated that the strong change in frequency content and amplitude of the surface acceleration due to liquefaction can be used to detect the occurrence of liquefaction. Recent centrifuge tests by Jafarian et al. [3] have shown a clear change in the rate of settlement and surface acceleration with the triggering of liquefaction. Bird et al. [4] recognised that damage can be generated through liquefaction-induced effects and ground shaking, and proposed a utility function to add the two causes based on the time at which liquefaction occurred. Kramer et al. [5] proposed a framework for assessing liquefaction effects (e.g. lateral spreading) based on ground motion intensity measures computed before and after the triggering of liquefaction. And recently Bouckovalas et al. [6], proposed a method for obtaining the surface shaking response spectra using the equivalent linear analyses of liquefied and non-liquefied deposits and taking the response from the pre and post liquefaction segments of the ground motion.

There are advanced nonlinear effective stress analysis techniques for evaluating the time of liquefaction. Unfortunately, these approaches require an extensive number of soil parameters, and non-trivial decisions about constraining the domain of the analysis (e.g. depth of the model). Whereas, simplified stress-based methods that quantify the soil liquefaction capacity in terms of the cyclic resistance ratio (CRR) (the amplitude of cyclic stress divided by the initial at rest vertical effective stress required to cause liquefaction under a certain number of cycles of equal stress amplitude), have been developed only for the assessment of liquefaction triggering, and often have biases or simplifications, that present significant drawbacks when used beyond their initial purpose (see Refs. $[7,8]$ ). The direct application of equivalent cycle counting methods (e.g. Ref. [9]), are considered to overcome some of the issues with estimating the time dependent cyclic demand but require the full stress time series and at least two parameters to define the CRR versus number of cycles relationship. Additionally, the typically application of these procedures applies the Palmer-Miner cumulative damage hypothesis which assumes a high number of cycles with essentially elastic behaviour, whereas liquefaction inherently involves a large change from initial elastic soil deformation behaviour, and therefore additional corrections should be applied to determine equivalent cyclic loading [10].

\footnotetext{
* Corresponding author.

E-mail address: maxim.millen@canterbury.ac.nz (M.D.L. Millen).
} 
Another possibility is to correlate liquefaction triggering with the cyclic strain amplitude (e.g. Ref. [11], as shear strain is strongly correlated to volumetric change [12]. While strain-based approaches can potentially be less influenced by soil fabric, ageing and confining stress, they still suffer from the same issues of the stress based methods, related to estimating the demand from a seismic wave. Alternatively, the dissipated energy of the soil during loading is closely linked to soil grain movement [13] and has been shown experimentally to be a loading independent measure of the liquefaction resistance of the soil (e.g. Refs. [14-19]). However, methods that adopt dissipated energy have two major drawbacks, one is that the estimation of the dissipated energy within a soil profile from a seismic shear wave is far from trivial, and very dependent on soil characteristics and shear strain [20]. Secondly, the dissipated energy rapidly increases as the soil approaches liquefaction (in tests with equal cycles of stress amplitude), and therefore a small change in the criteria for liquefaction triggering (e.g. change the limiting of excess pore pressure ratio from 0.95 to 0.98 ), can have a large impact on the evaluated capacity, though adjustments can be made to compute a dissipated energy under equivalent 'total stress' conditions that removes this effect [10].

To overcome some of the drawbacks of existing frameworks, this paper presents an energy based approach for estimating the time to liquefaction. The proposed method uniquely uses the cumulative absolute change in strain energy, since it is directly related to kinetic energy and has been shown to be uniquely related to liquefaction triggering. The paper also presents a novel equation for the calculation of the energy applied to a point by a travelling wave, a procedure for the calculation of the cumulative absolute change in strain energy from a broadband seismic motion and an estimation of the build up excess pore pressure and triggering of liquefaction with respect to time, throughout the depth of a soil deposit based on the upward propagating shear wave.

\section{Liquefaction capacity using the cumulative absolute strain energy}

\subsection{Definition}

The normalised cumulative absolute (change in) strain energy (NCASE) (or CASE when not normalised), which is calculated as the cumulative change in absolute peak strain energy divided by the at rest initial vertical effective stress (Equation (3)), is graphically represented as the sum of the absolute change in strain energy between the strain energy peaks in the response (Fig. 1). The peak strain energy points $(j)$ (local maxima and minima) can be determined graphically, or as the intercepts of the derivative shear strain using Equation (1), where $i$ is the incremental measurement of each parameter and $\Delta \gamma_{i}$ is the change in shear strain between the ith measurement and the one before it. Note that before applying Equation (1) the derivatives equal to zero should be removed from the time series to avoid flat peaks. The NCASE can then be calculated as the average absolute stress between peaks (Equation (2)) multiplied by the change in strain between the peak points divided by

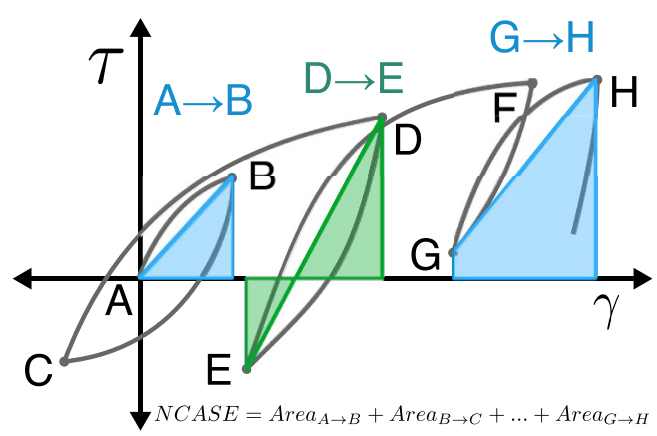

Fig. 1. Graphical calculation of cumulative absolute strain energy. the vertical effective stress, using Equation (3). The NCASE required to cause liquefaction can be identified as $N C A S E_{\text {liq }}$ and the NCASE generated by a loading (e.g. ground motion) can be identified as NCASE* .

peak $_{j}=\Delta \gamma_{i} \cdot \Delta \gamma_{i+1}<0$

$\left|\tau_{a v . j}\right|= \begin{cases}\frac{\left|\tau_{j+1}+\tau_{j}\right|}{2} & \tau_{j+1} \cdot \tau_{j} \geq 0 \\ \frac{\tau_{j+1}^{2}+\tau_{j}^{2}}{2 \cdot\left|\tau_{j+1}-\tau_{j}\right|} & \tau_{j+1} \cdot \tau_{j}<0\end{cases}$

$N C A S E=\frac{\sum_{j=0}^{n_{\text {peaks }}}\left|\tau_{a v \cdot . j}\right| \cdot\left|\gamma_{j+1}-\gamma_{j}\right|}{\sigma_{v, 0}^{\prime}}$

\subsection{Experimental validation}

Kokusho [18] investigated the undrained cyclic triaxial test data from Kokusho et al. [21] of reconstituted Futtsu beach sand at different relative densities and different percentages of fines content and demonstrated that a unique relationship existed between normalised dissipated energy and NCASE. The normalised dissipated energy was calculated as the area enclosed within the hysteresis loops divided by the at rest initial vertical effective stress as indicated in Equation (4). In the same paper it was shown that the dissipated energy required to liquefy the soil was load amplitude independent (corroborating efforts by other researchers on different soils (e.g. Refs. $[15,17]$ ) and by deduction, demonstrating that the NCASE required to liquefy the soil $\left(N C A S E_{\text {liq }}\right)$ is also load amplitude independent.

$\sum \frac{\Delta E_{\text {diss }, i}}{\sigma_{v, 0}^{\prime}}=\frac{\left(\tau_{i+1}+\tau_{i}\right) \cdot\left(\gamma_{j+1}-\gamma_{j}\right)}{2 \sigma_{v, 0}^{\prime}}$

To provide further evidence that $N C A S E_{\text {liq }}$ is load amplitude independent for different soils, the direct cyclic simple shear test data presented in Viana Da Fonseca et al. [22] were evaluated. The selected tests used in this study used two soils (Algeria Sand and Coimbra Sand), which were prepared by moist tamping at different relative densities (15 to $75 \%$ ) and tested saturated at different confining stresses (100 to $300 \mathrm{kPa}$ ) and across a range of cyclic stress ratios (0.04-0.36). More information regarding the description of the tests can be found in Ref. [22]. The criteria for liquefaction was set at the vertical stress reducing to less than $8 \mathrm{kPa}$ or the single amplitude shear strain exceeding $3.75 \%$, consistent with the criteria from Viana Da Fonseca et al. [22]. The results for all tests that reached the liquefaction criteria between 2 and 40 cycles, and the transient excess pore pressure ratio $\left(r_{u}\right)$ (excess pore pressure divided by the initial vertical effective stress) reached $80 \%$, are shown in Fig. 2. Fig. 2a) shows the conventional definition of liquefaction resistance in terms of number of cycles $\left(n_{\text {cycles }}\right)$ shown on the $y$-axis as the dependent variable compared to the applied cyclic stress ratio (CSR). Fig. 2b), c) and d) show that the normalised dissipated energy, the $N C A S E_{\text {liq }}$, and the ratio between them have a low dependance on load amplitude, consistent with findings from Kokusho [18]. These tests also demonstrate that the $N C A S E_{\text {liq }}$ can be obtained through element tests or correlations with the dissipated energy capacity, while further research is required to obtain $N C A S E_{\text {liq }}$ from in-situ tests (e.g. cone penetration test).

\section{Estimation of accumulated strain energy at depth of interest}

In the previous section the triggering of liquefaction was quantified using $N C A S E_{\text {liq }}$. In this section an exact solution to estimate the NCASE demand $\left(N C A S E^{*}\right)$ at any depth from a free surface will be developed for an upward propagating broadband seismic shear motion.

The cumulative absolute kinetic energy (CAKE), can be computed using Equation (5) as the total kinetic energy per unit volume given and taken from a point. Equation (5) is simply the sum of the cumulative 


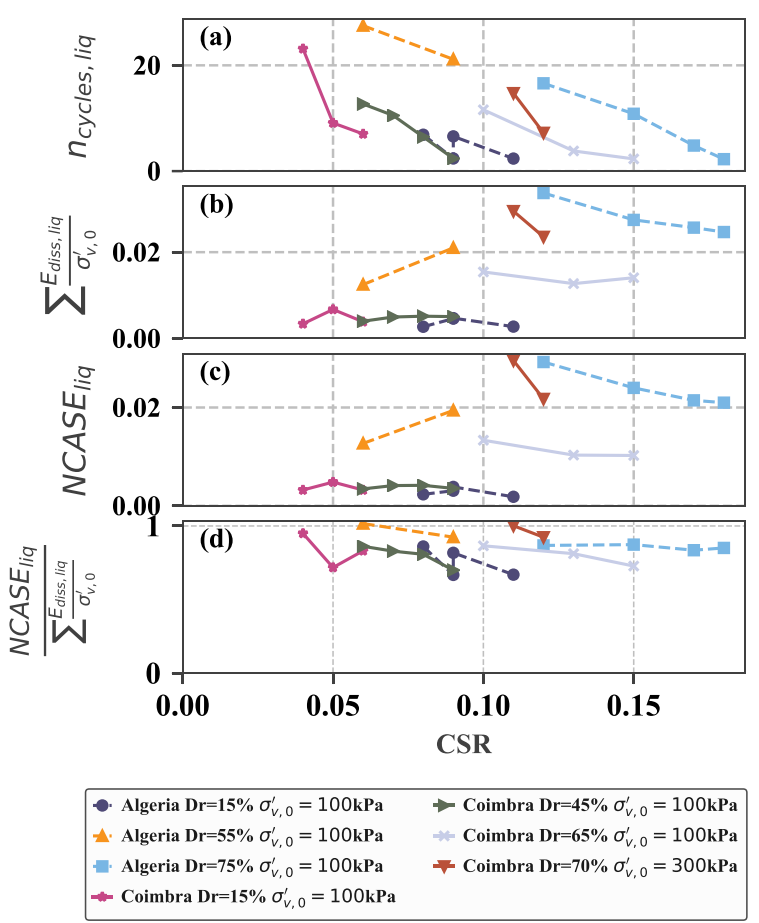

Fig. 2. Experimental validation that the NCASE required to liquefy the soil is load amplitude independent.

absolute change in kinetic energy or for a continuous function can be computed as the integral of absolute power. The naming is consistent with other intensity measures such as cumulative absolute velocity, which is computed as the integral of absolute acceleration. As a ground motion intensity measure it can be computed as the unit cumulative absolute kinetic energy (UKE), where the soil mass density ( $\rho$ ) is taken as 1.0. Note that the calculation of UKE requires properly baseline corrected records, as a drift or constant velocity influences the calculation of the change in energy.

$C A K E=\rho \cdot \sum_{i=1}^{n} \Delta\left(\dot{u}_{i} \cdot\left|\dot{u}_{i}\right|\right)=\rho \cdot \int\left|\frac{d \dot{u}_{i}{ }^{2}}{d t}\right| d t$

For a wave moving in an infinite elastic medium the kinetic energy and strain energy are each $50 \%$ of the total travelling wave energy [23]. For a point in that infinite medium, the kinetic and strain energy are zero prior to the wave reaching the point, and zero after the wave has completely passed through, and the sum of all of the absolute changes in the kinetic energy experienced at that point is equal to the sum of the absolute changes in strain energy, representing the total absolute work done by the propagating motion on the soil. The CAKE from a propagating wave in a 3D space from a source (i.e. an earthquake) can then be predicted through the principle of conservation of energy and using simple geometric relationships to account for radiation damping. However, the strain in a soil near the ground surface of a one-dimensional soil profile is caused by the energy from the upward and downward propagating shear waves and therefore the simple one-to-one relationship between CAKE and CASE is no longer applicable near a free surface. In fact, at some depths, the strain energy is highly frequency dependent and not at all proportional to the kinetic energy. There are several existing energy based methods (e.g. Refs. [15,24,25], however, these existing methods assume that the strain energy is linearly proportional to the upward propagating kinetic energy density [26] or in the case of [27]; proportional to Arias Intensity [28]. The energy density proposed by Sarma [26] is the integral of the squared velocity multiplied by the soil density and shear wave velocity, and is a measure of the total wave energy, unlike CASE which is the work done by the wave on a point of soil as it passes through it (see Section 3.5 for further comparison). Furthermore, clearly the assumption of linearly proportional is not suitable near a free surface as, at the surface the ground motion amplitude doubles as the upward and downward waves are in phase across all frequencies [26], and the kinetic energy tends to four times the upward kinetic energy, while the strain energy tends to zero. Conversely, at great depths, where there is no surface reflection (approximately an infinite medium), the strain energy and kinetic energy become equal. While at intermediate depths the CASE depends on the frequency content of the propagating motions. Additionally, in heterogeneous soil deposits waves reflect off the surface and off stiffer mediums, and the soil can experience the wave energy numerous times.

\subsection{Energy of sine waves}

To emphasise the importance of the frequency content on the CASE with depth, a simple linear viscoelastic one-dimension site response study was conducted using the python-based site response analysis package, Pysra, [29]. The soil profile was $50 \mathrm{~m}$ in height with a shear modulus of $30 \mathrm{MPa}$, viscous damping of $3 \%$ and unit weight of $17.5 \mathrm{kN} / \mathrm{m}^{3}$. Fig. 3a shows the calculated energy throughout the depth of the soil profile for a sine wave of frequency equal to $3.0 \mathrm{~Hz}$, where the cumulative energy has been normalised by the CAKE of the input motion (Equation (5)) and the depth has been normalised by the wavelength $(\lambda)$. It can be clearly seen that CASE and CAKE are offset by a quarter of a wavelength but both develop maxima and minima at intervals of half a wavelength, consistent with analytical work by Ref. [30]. The interaction is strongest at the surface and less cancelation occurs at greater depths as the downward energy decreases in relation to the upward energy due to damping. Note that the upward CAKE at the base is equal to the input CAKE and due to damping it decreases to approximately $45 \%$ at the surface. Sine waves are a widely used input motion for numerical and centrifuge studies on liquefaction, however, the strong variation in strain energy with depth that is observed for single frequency sine waves suggests they are not suitable input motions for some studies of liquefaction behaviour.

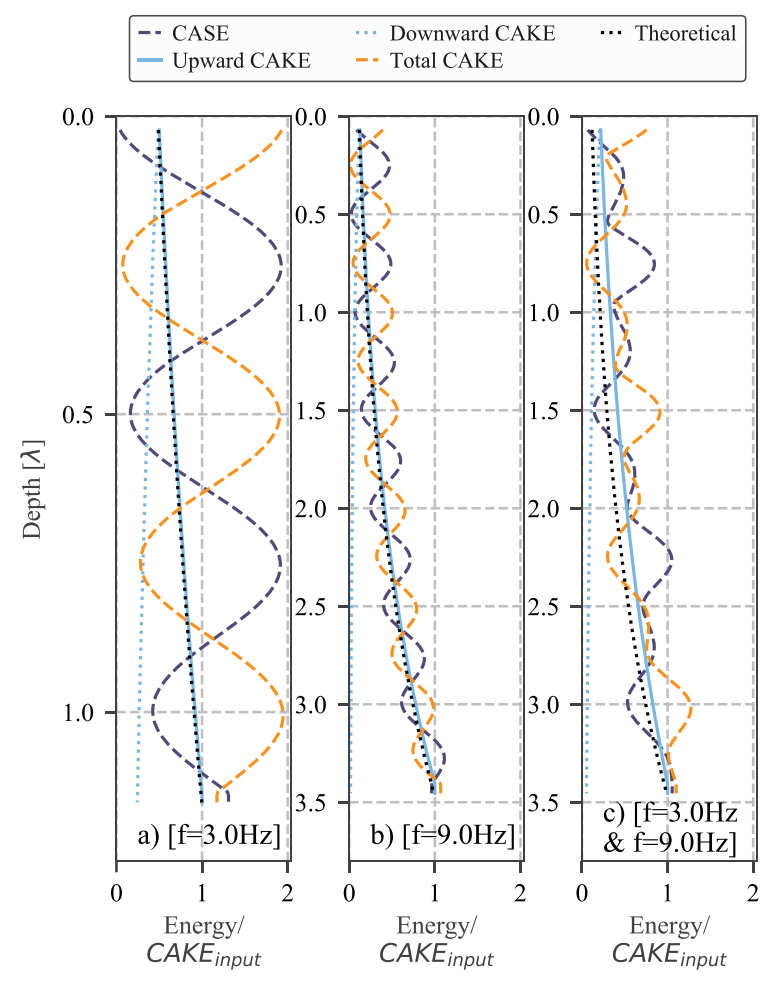

Fig. 3. Energy of sine waves in homogenous soil deposits. 
Fig. $3 \mathrm{~b}$ shows the energy from a sine wave with a frequency of $9 \mathrm{~Hz}$. The increased frequency means the wave energy decreases much more rapidly. The upward CAKE can be seen to decrease to nearly $10 \%$ at the surface and the downward kinetic energy approaches zero at a depth of five wavelengths. The theoretical solution for the reduction in energy of a travelling wave due to viscous damping (Equation (6)) is also shown to exactly match the upward CAKE, and is a useful reference point for estimating the energy of seismic motions. Because the downward energy tends to zero at the base of the domain, the interaction tends to zero, and the total kinetic energy and the strain energy tend to the input kinetic energy.

Fig. 3c show the propagation of a two frequency motion, made of a sine wave at $3 \mathrm{~Hz}$ and a second sine wave with three times the amplitude at $9 \mathrm{~Hz}$. The wavelength and theoretical reduction are shown for the frequency of $9 \mathrm{~Hz}$. The reduction differs slightly from the observed reduction since the energy of the lower frequency wave reduces at a slower rate. The response is essentially a superposition of the two previous cases and some of the full cancellations of energy observed in the previous cases now experience some strain energy, while when two minima combine, the cancellation is still almost to zero. Most importantly for liquefaction assessment, the strain energy is higher near the surface compared to the low frequency case (a), and does not experience the cancellation at a quarter of a wavelength observed in the high frequency case (b). Thus more strain energy occurs near the surface of the soil deposit.

Energy $_{z}=$ Energy $_{[z=H]} \cdot \exp \left(-\xi \cdot z / V_{s} \cdot 2 \cdot \pi \cdot f_{1}\right)^{2}$

\subsection{Surface energy spectra}

For real ground motions the behaviour is more complex due to the large variation in frequency content. To allow an accurate prediction of the CASE at any depth, a novel spectrum has been developed called the nodal surface energy spectrum (NSES) to give an exact solution to the CASE for any broadband seismic shear wave reflecting off a perpendicular free-surface in an elastic homogenous deposit. The NSES is a unique parameter of the ground motion, and is calculated independently from the soil and site characteristics. It characterises how an incident wave interacts with its reflected self based on some time shift, corresponding to the time taken to travel to the free-surface and return to the point of interest.
The calculation of the NSES is outlined in Fig. 4, where the reflected acceleration time series $\left(\ddot{u}_{i+s h i f t}\right)$ is equal to the incident motion $\left(\ddot{u}_{i}\right)$, except that it is shifted backwards in time (padded with zeros at the start of the time series), where the time shift is equal to two times the travel time from the surface to the point of interest (Fig. 4b). The incident and reflected waves are then subtracted to obtain the time shifted motion $\left(\ddot{u}_{t s, i}\right.$ ) (Fig. 4c), where scale factors $(A$ and $B$ ) can be applied to the incident and reflected motion in Equation (7) to represent reduction in amplitude due to damping. The calculation of UKE using Equation (5) of the time shifted motion, is equal to the CASE at the point of interest (Fig. 4d) and can be repeated for different travel times to obtain a spectrum (Fig. 4e). When the travel time exceeds half the length of the record, then the CASE is equal to two times the CAKE of the upward motion, since the upward and downward motions do not interact and there is zero damping. Note that if the two motions were added instead of subtracted then the UKE of the time shifted motion would be equal to the CAKE, since at a free surface the incident and reflected acceleration amplitudes interact constructively. The solution can also be applied to reflections off a rigid surface, where amplitudes interact destructively. In this situation, the CASE would be computed using the addition of the two waves, while the CAKE would be obtained with the subtracted waves.

$\ddot{u}_{t s, i}=A \cdot \ddot{u}_{i}-B \cdot \ddot{u}_{i+s h i f t}$

In the time domain Equation (7) can only provide an exact solution for an elastic homogenous soil profile that is undamped or for a viscoelastic soil profile with a harmonic input motion, where the exact frequency dependent damping and energy reduction can be obtained using Equation (6). However, in the following sections the NSES solution is shown to provide a suitable solution for layered, liquefying soil deposits.

A clear advantage of using the principle of conservation of energy to obtain demand, rather than the 'rigid-body' assumption adopted in simplified stress based procedures (e.g. Ref. [31]) is that the demands that are calculated with depth correctly reflect the frequency content of the ground motion. The strain energy computed using the NSES spectrum method can also be used to calculate stresses, strains and dissipated energy through simple assumptions of the hysteretic behaviour of the soil and could therefore be used to directly replace the stress reduction factor in simplified stress based procedures (e.g. Ref. [31]).
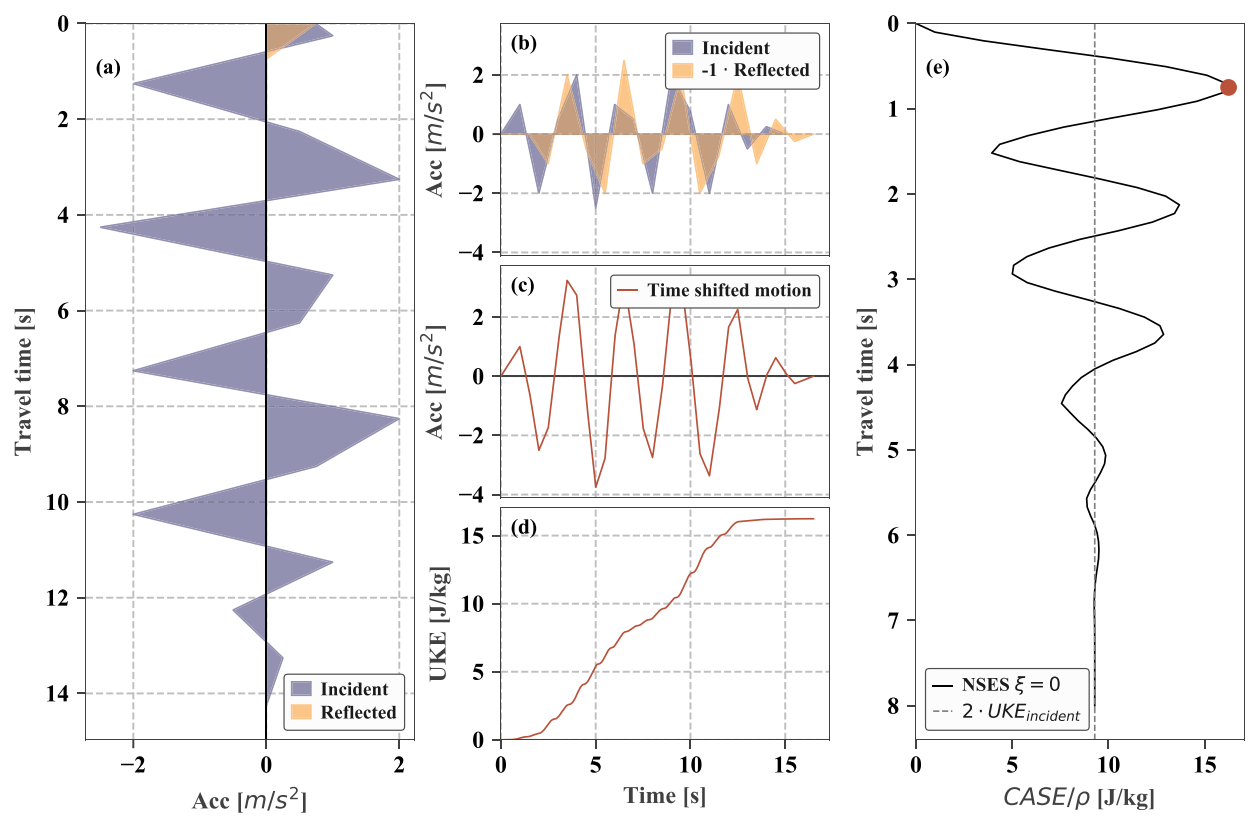

Fig. 4. Steps involved in the computation of the nodal surface energy spectrum (NSES). 


\subsection{Damped surface energy spectra}

Fig. 5 shows the response of different motions for a $50 \mathrm{~m}$ homogeneous linear soil profile with a $20 \mathrm{MPa}$ shear modulus and unit weight of $17.5 \mathrm{kN} / \mathrm{m}^{3}$. A $5 \mathrm{~Hz}$ sine wave motion is shown in Fig. 5, along with the 'theoretical CASE', estimated using the NSES method and Equation (7) where the reduction factors $A$ and $B$ were computed by Equation (6) using the $5 \mathrm{~Hz}$ input frequency. As expected, the theoretical solution provides an indistinguishable match to the CASE from the simulation.

Unfortunately, by definition a broadband motion has multiple frequencies and therefore an exact solution cannot be obtained for a soil profile with viscous damping using Equation (7). Instead the damped spectrum $\left(N S E S_{\xi, z=h}\right)$ can be approximated using Equation (8) by applying a damping reduction factor $\left(\eta_{t}\right)$ as a function of height (or travel time) to the undamped spectrum $\left(N S E S_{\xi=0}\right)$. Where $\eta_{t}$ is estimated as a linear reduction from the point where the upward motion is defined (base of soil profile) to the surface using Equation (9). Where $t_{h}$ is the travel time from the surface $(z=0)$ to the point of interest $(z=h), t_{H}$ is the travel time from the surface to the base of the soil profile and $\eta_{\text {total }}$ is defined in Equation (10) as the ratio of the CAKE of the upward propagating shear wave at the surface compared to the upward CAKE at the base of the profile computed using Equation (6), with a chosen representative vibration frequency.

$N S E S_{\xi, z=h} \approx \eta_{t} \cdot N S E S_{\xi=0}$

$\eta_{t}=\eta_{\text {total }}+\left(1-\eta_{\text {total }}\right) / 2 \cdot t_{h} / t_{H}$

$\eta_{\text {total }}=C A K E_{\text {up }, z=0} / C A K E_{u p, z=H}$

The NSES solution with and without viscous damping is shown for two recorded motions (motion 770 - Loma Prieta (1989) earthquake, recorded at Gilroy Array 3 station; motion 5263 - Chuetsu-oki (2007) earthquake, recorded at NIG017 station from the NGA2West database [32]) in Fig. 5, where the representative frequency was optimised to reduce the error in the CASE estimate. The 'predicted CASE' shows an exact match for the zero viscous damping situation and a minor

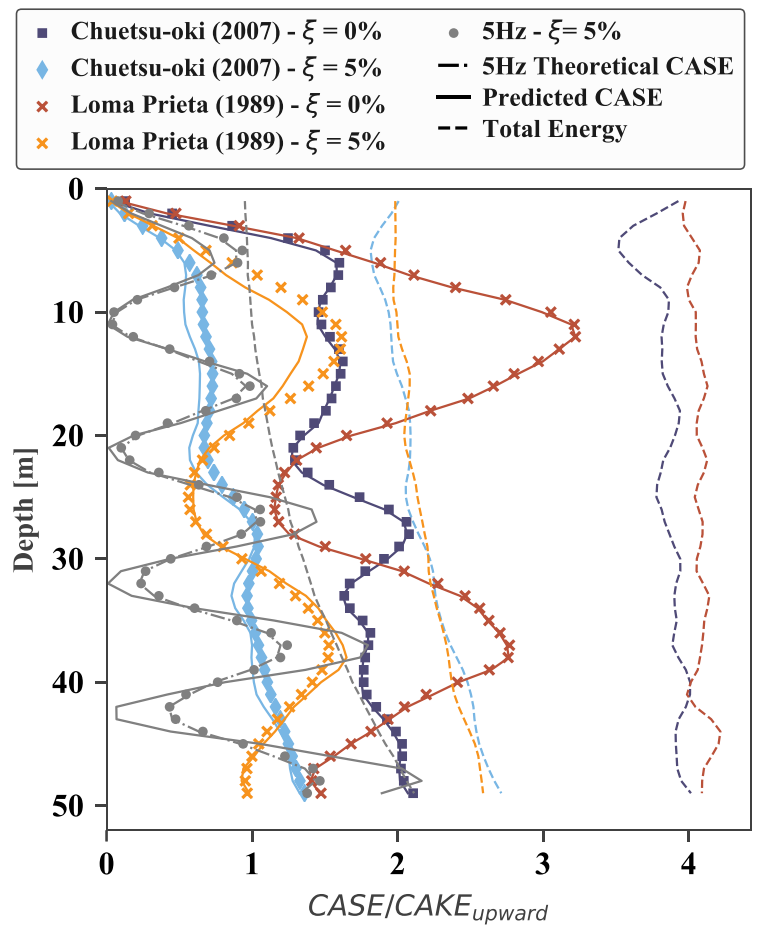

Fig. 5. Strain energy in damped and undamped linear homogeneous soil profiles versus NSES method. variation for the approximated solution using the damped NSES method. Although difficult to discern from Fig. 5, the relative error in the prediction for the Chuetsu-oki motion increases near the surface. The increase in relative error is partly because the CASE tends to zero and therefore magnifies the relative error, and partly because the CASE near the surface is dependent on high frequency content, which is strongly reduced up the viscoelastic profile. However, it should be noted that soil is not a true viscoelastic material and typically high frequencies are over damped when using constant damping across all frequencies [33]. The approximate damped NSES solution is also demonstrated for the sine wave input, here the biases due to the simplification are more apparent, where an under-prediction is observed near the surface and over-prediction at depth. This bias is smoothed in the broadband solution due to it being frequency dependent.

The total wave energy (CASE plus CAKE) computed for each analysis is also plotted in Fig. 5 in dashed lines. It can be seen that for the undamped instances, the total energy remains constant at about four times the input CAKE (since the energy is reflected off the ground surface, the soil experiences the total wave energy twice). While the reference value of four is a useful benchmark, it does not apply in the case of layered soil, where waves are reflected and a soil layer may experience the same wave energy numerous times. For the damped case, the total energy of the sine wave is consistent with the reduction from Equation (6), where the upward and downward waves are first reduced and then added together. Finally it should be noted that the damped solution allows for the surface motion to be used as the incident motion to determine the strain energy at depth, however, since the surface motion has been reduced due to travelling through the deposit, the damping reduction should therefore be greater than one and can be computed with Equation (11).

$\eta_{t, \text { surf. }}=1+\left(1-\eta_{\text {total }}\right) / 2 \cdot t_{h} / t_{H}$

\subsection{Strain energy in layered soil}

For a layered soil profile a change in impedance causes wave energy to be reflected. Since the NSES only represents the interaction of a wave hitting a free-surface in a homogenous half space, the reflected energy is not captured. Also a reduction in shear wave travel velocity means the wave can perform more work on the soil as it passes through. Fig. 6 shows three simple yet challenging hypothetical cases for predicting the CASE in a layered deposit, compared to linear analysis with the Pysra package. The ground motion used for all three analyses was the Bursa Tofas station record from the 1999 Magnitude 7.5 Earthquake in Kocaeli Turkey. Fig. 6a shows two layers where the top $45 \mathrm{~m}$ has a shear modulus of $10 \mathrm{MPa}$ while in the lower $15 \mathrm{~m}$ layer the shear modulus is $30 \mathrm{MPa}$, the unit weight for both layers was $20 \mathrm{kN} / \mathrm{m}^{3}$. A large viscous damping of $15 \%$ was set for both layers to remove the energy content of the downward wave. The CASE from the linear analysis, $C A S E_{\text {linear, }}$, is clearly amplified across the layer boundary by approximately a factor of

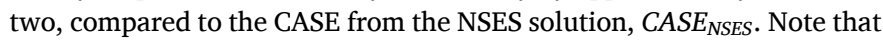
for a travelling wave in an infinite medium this amplification would reach a maximum ratio of four as the impedance contrast increases. Fig. $6 \mathrm{~b}$ shows a contrast in unit weight from $10 \mathrm{kN} / \mathrm{m}^{3}$ for the first $45 \mathrm{~m}$ and $20 \mathrm{kN} / \mathrm{m}^{3}$ for the last $15 \mathrm{~m}$. The shear modulus was $30 \mathrm{MPa}$ and the viscous damping was $15 \%$ for the whole soil profile. The NSES provides a reasonable prediction even though the CAKE changes across the boundary. The third soil profile shown in Fig. $6 \mathrm{c}$ is of a $15 \mathrm{~m}$ layer of $10 \mathrm{MPa}$ shear modulus at $30 \mathrm{~m}$ depth, surrounded by $30 \mathrm{MPa}$ soil, all layers have $20 \mathrm{kN} / \mathrm{m}^{3}$, and $5 \%$ damping. In this scenario waves are reflected off the both soil interfaces plus the surface, and the interaction becomes very complex, ultimately the $C A S E_{\text {linear }}$ is almost three times larger than the $C A S E_{N S E S}$ value.

The exact level of amplification is highly dependent on the level of soil energy dissipation and the stiffness contrast across the layers above and below and requires an in-depth study to quantify for different 


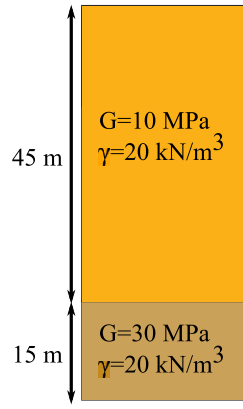

a)

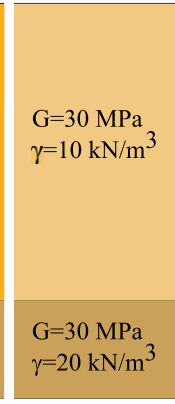

b)

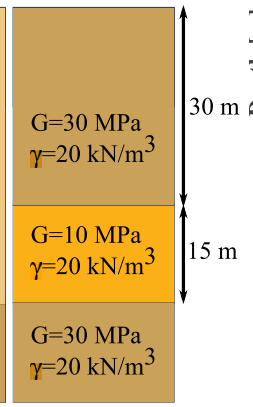

c)

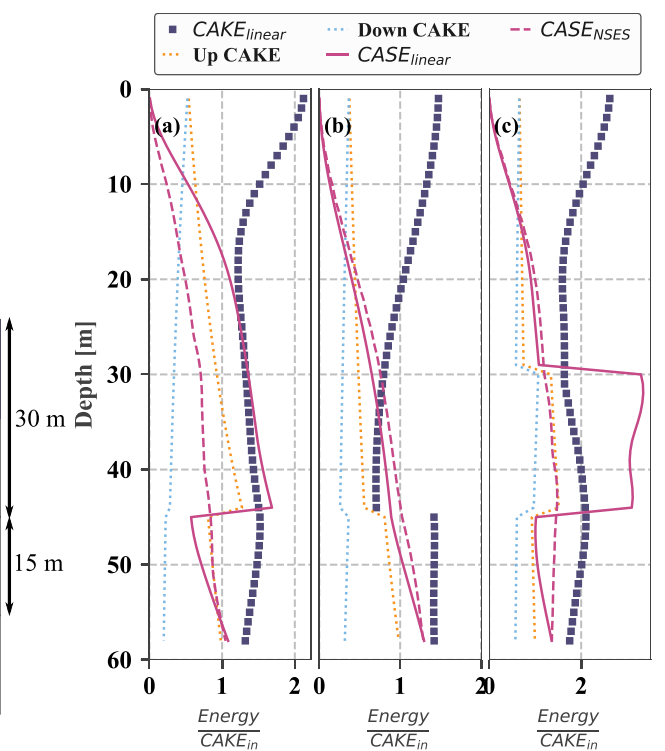

Fig. 6. Strain energy in layered soil profiles versus NSES method.

situations. For the validation of the NSES method in the remainder of this paper the amplification for a soil layer between two stiffer layers will be approximated through a simple scaling of the homogenous solution using Equation (12). Where CASE $E_{\text {uniform }}^{*}$ is the strain energy as if the soil was uniform, $G_{\text {layer }, k}$ is the shear modulus of the kth layer, $G_{\text {input }}$ is the shear modulus where NSES of the upward motion was computed (i.e. the surface or base of the deposit).

$C A S E_{\text {laye }, k}^{*}=\operatorname{CASE}_{\text {uniform }, i}^{*} \cdot \frac{G_{\text {laye }, k}}{G_{\text {input }}}$

\subsection{Estimation of energy spectra}

The NSES method provides a design and assessment tool for liquefaction that is analogous to the design and assessment of buildings using the SDOF elastic response spectrum. The exact solution can be obtained for a true elastic SDOF, and the inelastic response of nonlinear multidegree-of-freedom systems is approximated through modification factors. To improve the usability of the NSES method for design and assessment where the exact ground motion is unknown, the spectrum should be quantified using simple relationships in the same manner as a typical SDOF design response spectrum.

Given that the key quantity for the estimation of liquefaction is the normalised CASE, the undamped NSES has been plotted in Fig. 7a divided by the travel time (a proxy for vertical effective stress) to illustrate some of the key characteristics. Since liquefaction assessment is typically only concerned with the top $30 \mathrm{~m}$ of soil, the spectrum only needs to be quantified in the first $2 \mathrm{~s}$ to cover shear wave velocities as low as $30 \mathrm{~m} / \mathrm{s}$. In this range the spectrum consists of three parts, a rapidly increasing section as frequencies become out-of-phase, up to the highest significant frequency, then a plateau to the dominant frequency, which corresponds to a linearly increasing CASE. The third, decreasing branch typically follows a 2 (travel time) relationship, which corresponds to a constant CASE, where the CASE is equal to two times the input CAKE, and the time shift causes approximately equal constructive and destructive interaction from the upward and downward wave. Damping would further decrease amplitude and could be approximated either through Equation (8) or possibly exact frequency domain solutions. The spectra share similar characteristics to the SDOF elastic response spectra (Fig. $7 \mathrm{~b}$ ), which has been plotted with double the period range since the travel time corresponds to double the time shift in the NSES method. The peaks are more erratic in the SDOF spectra as
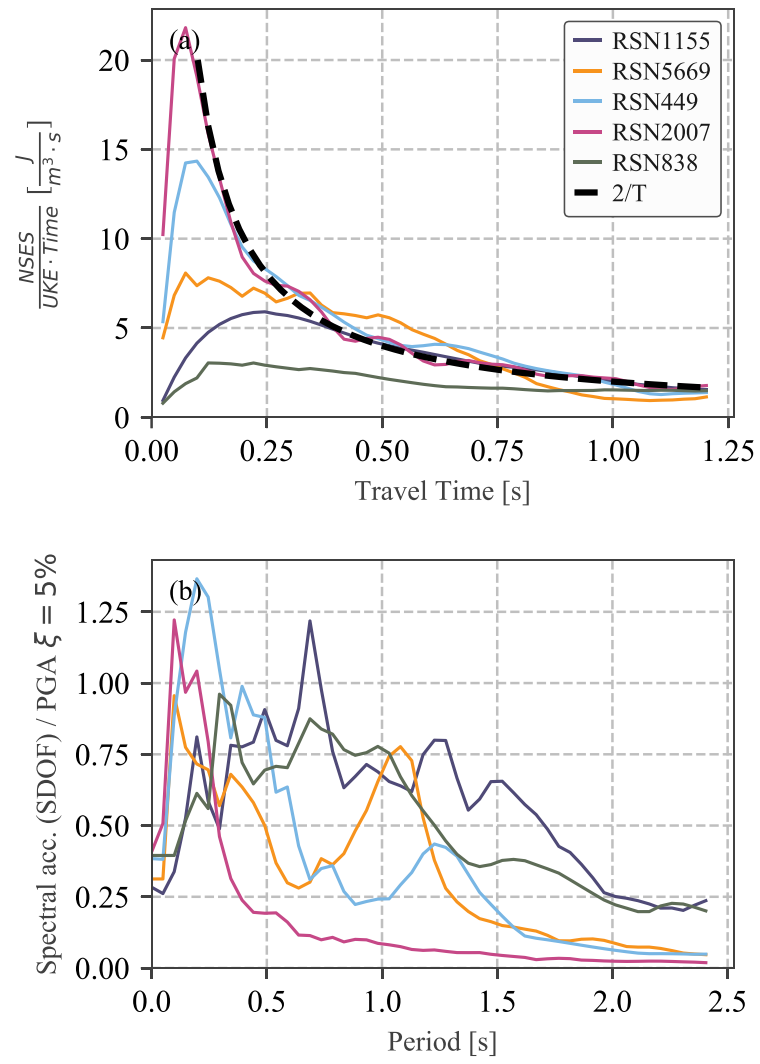

Fig. 7. Comparison of NSES and SDOF response spectrums.

expected, since it is a measure of an instantaneous single value and therefore more sensitive to changes in the response period for a broadband motion. The amplification is more pronounced in the NSES, since the energy quantity is proportional to the velocity squared. While further work is needed to characterise the NSES, the key frequencies of the NSES are directly linked to the site characteristics and magnitude of the earthquake and therefore can be simplified for design and assessment purposes.

The NSES method also provides a useful tool for advanced effective stress analysis in a performance-based design and assessment context, 
where the selection of ground motions should best reflect the likely seismic hazard with low variability. The average energy for the travel times corresponding to critical liquefiable layer should present a highly sufficient measure for ground motion selection or selection using a conditional mean spectrum [34] centred around the critical depth. The use of CASE, which is a spectral, cumulative parameter, adequately accounts for the frequency, duration and amplitude aspects of the seismic demand for liquefaction triggering from an upward propagating shear wave. Unfortunately, to the best knowledge of the authors, there are no prediction equations available for UKE or for the NSES spectral coordinates.

The UKE is not the integral of the squared velocity (ISV) (Equation (13)) or "energy density", that has been widely used in other liquefaction estimation procedures (e.g. Ref. [18]) and used for the computation of the energy magnitude [35]. The UKE shares similarities to this and other widely used intensity measures, however, the key difference is that the CAKE is the integral of the rate of change of kinetic energy with time, whereas the energy density is the integral of kinetic energy with time. The two expressions provide the same value for a sine wave of oscillation frequency, $f_{1}$, where the energy density is computed over a small increment of distance $(d z)$ for a soil of shear wave velocity $V_{s}$, such that $d z=V_{s} /\left(4 \cdot f_{1}\right)$ but for a broadband motion, there is no exact compatibility.

Fig. 8 shows the intensity measure relationships for a simple harmonic oscillator with unit amplitude and varying frequency in (a), and $1 \mathrm{~Hz}$ and varying amplitude in (b). The ratio of ISV over UKE is inversely proportional to the frequency by a factor of 4 and independent of the amplitude whereas the ratios for cumulative absolute velocity (CAV) (Equation (14)) and Arias Intensity $\left(I_{a}\right)$ (Equation (15)) over UKE scale linearly with frequency, while Arias Intensity is constant with amplitude and CAV is inversely proportional. Equations (13)-(16) have all been written with respect to the velocity time series $(\dot{u})$ for direct visual comparison, with $d t$ being the time step.

$I S V=d t \cdot \sum \dot{u}_{i}{ }^{2}$
$C A V=\sum\left|\Delta \dot{u}_{i}\right|$
$I_{a}=\frac{\pi}{d t \cdot g} \sum\left(\Delta \dot{u}_{i}\right)^{2}$
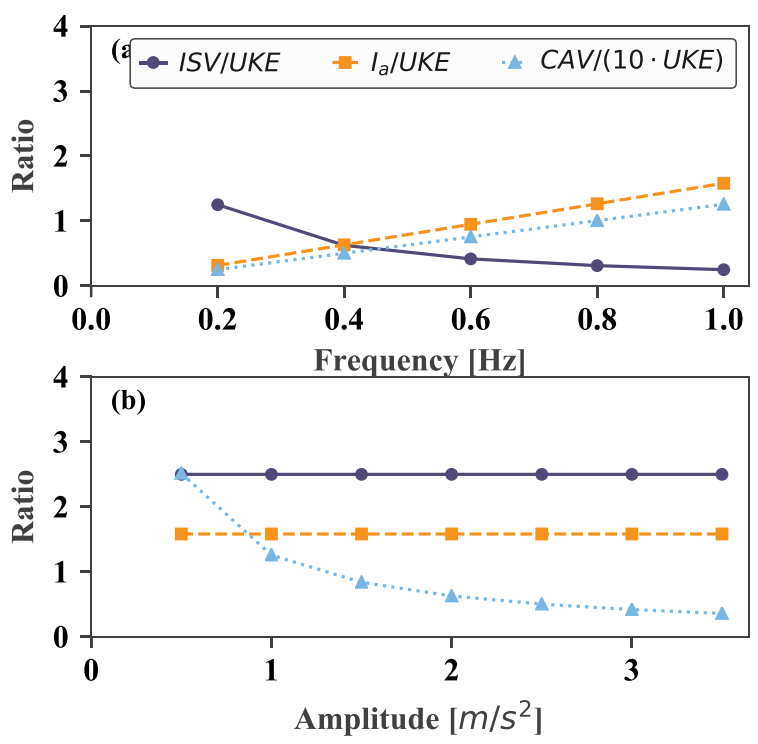

Fig. 8. Unit kinetic energy vs other intensity measures.
$U K E=\sum_{i=1}^{n} \Delta\left(\dot{u}_{i} \cdot\left|\dot{u}_{i}\right|\right)$

For the purpose of immediate use, a set of ground motions can be conditionally selected based on the earthquake magnitude, distance and either expected cumulative absolute velocity after application of $5 \mathrm{~cm} / \mathrm{s}^{2}$ threshold acceleration (CAV5) [36] or Arias Intensity. Both CAV5 and Arias Intensity have been shown to be closely linked to liquefaction triggering, see Refs. [36,37]; and prediction equations in Refs. [36,38]; respectively.

The NSES of these records can then be computed and applied to the outlined procedure. Given the relationships observed in Fig. 8, the ground motions should be used unscaled to avoid unintended biases.

\section{Numerical validation study}

A series of linear, equivalent linear, nonlinear total stress and nonlinear effective stress one dimensional analyses were used to validate the NSES method for the estimation of NCASE* and ultimately estimate the build up of excess pore pressure and triggering of liquefaction.

The NSES method used the upward motion at the base, the elastic soil properties and 3\% damping, and Equation (12) for the impedance. Five hundred synthetic soil profiles were randomly generated using the ranges and properties outlined in Table 1, and each soil profile was simulated using all analysis types. The soil profiles consisted of two clay layers (top and bottom) and a middle layer of sand (Fig. 9). The water table was assumed at the interface of the first and second layers. For layers one and three, one random number was generated for each layer to sample the cohesion, shear modulus and void ratio, to account for the correlation between these parameters. By keeping the same ratio between these parameters the soil could be considered as a clay with a

Table 1

Soil profile inputs.

$G_{0}=167 \cdot \sqrt{N_{1,60}+2.5} \cdot R[0.7,1.5]$

$G=G_{0} \cdot p_{a t m} \cdot \sqrt{p^{\prime} / p_{a t m}}$

\begin{tabular}{ll}
\hline Parameter & Range \\
\hline Height of L1, $H_{1}$ & {$[0.5-8.5] \mathrm{m}$} \\
Height of L2, $H_{2}$ & {$[0.5-10] \mathrm{m}$} \\
Total profile height, $H_{\text {total }}$ & {$\left[20-\mathrm{max}\left(2.5 \cdot\left(H_{1}+H_{2}\right), 30\right)\right] \mathrm{m}$} \\
Permeability of L1, $k_{1}$ & $8 \cdot 10^{-8} \mathrm{~m} / \mathrm{s}$ \\
Permeability of L2, $k_{2}$ & $1.6 \cdot 10^{-5} \mathrm{~m} / \mathrm{s}$ \\
Permeability of L3, $k_{3}$ & $10^{-9} \mathrm{~m} / \mathrm{s}$ \\
Dilatancy, $\psi$ & $0^{\circ}$ \\
Properties of layer 2 & \\
Specific gravity, $G_{s}$ & 2.65 \\
Poisson ratio, $\nu$ & 0.3 \\
Critical friction angle, $\varphi$ & $33^{\circ}$ \\
Minimum void ratio, $e_{\min }$ & 0.5 \\
Maximum void ratio, $e_{\max }$ & 0.8 \\
Relative density, $D_{r}$ & {$[0.3-0.8]$} \\
Normalised SPT, $N_{1,60}$ & $46 \cdot D_{r}^{2}$ \\
Normalised shear modulus, $G_{0}$ & Equation $(17)[\mathrm{Pa}]$ \\
Other properties of layers 1 and 3 & {$[180-200] \mathrm{kPa}$} \\
Poisson ratio, $\nu_{1}$ & {$[0.6-0.8]$} \\
Specific gravity, $G_{s}$ & 0.4 \\
Undrained strength of L1 & $1000 \cdot 0.7]$ \\
Undrained strength of L3 & \\
Void ratio of L1 & 2.7 \\
Void ratio of L3 & {$[30-34] \mathrm{kPa}$} \\
Initial shear modulus, $G_{i}$ & strength \\
\hline & \\
& \\
&
\end{tabular}




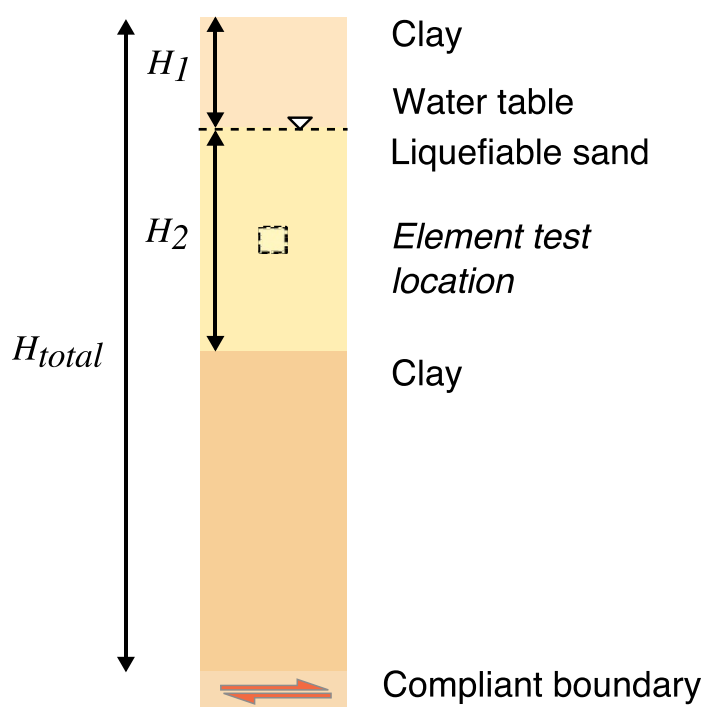

Fig. 9. Soil profile definition and numerical element test location.

plasticity index of $30 \%$. For the sand layer, the relative density $\left(D_{r}\right)$ was randomly sampled between $30 \%$ and $80 \%$ and used to calculate an equivalent clean sand normalised SPT blow count $\left(N_{1,60, c s}\right)$ as $46 \cdot D_{r}$. The stress dependent shear modulus $(G)$ was defined as in Equation (18) using the normalised shear modulus $\left(G_{0}\right)$ calculated from Equation (17) from Ref. [39] with an additional random multiplier between 0.7 and 1.5 .

The FLAC 8.0 software [40] was used to perform the total and effective stress analyses. The crust and base non-liquefiable layers were modelled with the Mohr-Coulomb model and the hysteretic damping option. The combination of the models meant that the strain related stiffness reduction would be captured with the hysteretic damping, while the Mohr-Coulomb model had a slightly lower yield criterion than the asymptote of the hysteretic damping and thus when the yield criterion was reached, the hysteretic damping became inactive in those zones, thus avoiding some numerical instabilities at large strains [41]. The hysteretic damping was set using the Default model in FLAC, using values $\mathrm{L} 1=-2.3 \mathrm{~L} 2=0.63$ to match the expression from Vardanega and Bolton [42] for plasticity index of $30 \%$ for all soils, since the ratio of initial shear modulus to undrained strength was the same for all soils.

For the liquefiable sand the PM4Sand constitutive model [39] was adopted to simulate the constitutive behaviour of the second sand layer in the dynamic phase. The model follows the framework of the stress-ratio controlled, critical state compatible, bounding surface plasticity model by Dafalias and Manzari [43] for sand. The use of FLAC and the PM4Sand model has been used to successfully simulate numerous laboratory element tests, centrifuge tests, and field case studies (e.g. Refs. [44-47]. The PM4Sand model requires the contraction rate parameter $\left(h_{p 0}\right)$ to be set and can be used to calibrate the model to a target CRR for building up excess pore pressure. This parameter was set using Equation (19) that was developed through a regression analysis where the $h_{p 0}$, relative density and shear modulus were systematically varied with a confining stress of $100 \mathrm{kPa}$, and the CRR for 15 cycles was obtained through numerical element tests. The target CRR for 15 cycles that was used to set the $h_{p 0}$ factor was calculated using Equation (20), which is the expression from Boulanger and Idriss [48] with an additional random coefficient $\left(c_{f}\right)$. The $c_{f}$ parameter was randomly sampled between a lower limit of 0.55 and an upper limit as the asymptote of Equation (19).

$h_{p 0}=\frac{\left(C R R_{n 15, \text { target }} \cdot\left(2.05-\left(2.4 \cdot D_{r}\right)\right)\right.}{\left(1-\operatorname{CRR}_{n 15, \text { target }} \cdot\left(12.0-\left(12.5 \cdot D_{r}\right)\right)\right)}$
$C R R_{n 15, \text { target }}=\exp \left(\frac{N_{1,60, c s}}{14.1}+\left(\frac{N_{1,60, c s}}{126}\right)^{2}-\left(\frac{N_{1,60, c s}}{23.6}\right)^{3}+\left(\frac{N_{1,60, c s}}{25.4}\right)^{4}-2.8\right) \cdot c_{f}$

For the total stress analyses, the bulk modulus of the water was set to zero to avoid the build-up of pore pressure, whereas for the effective stress analyses it was set to $2.2 \mathrm{GPa}$. An additional $2 \%$ Rayleigh damping was specified at $0.56 \mathrm{~Hz}$ and $2 \mathrm{~Hz}$ to mitigate numerical instability. The base of the soil profile was modelled as a 'compliant base' (non-rigid base), providing a silent non-reflecting boundary. Therefore the motions were input at the base as a stress $\left(\tau_{i n}\right)$ using Equation (21) [41], where the stress factor $\left(k_{\tau}\right)$ was set at 1.1 for all analyses, and soil mass density $(\rho)$ and shear wave velocity $\left(V_{s}\right)$ were for the bottom layer and $\dot{u}$ was the velocity time series of the outcrop motion. The FLAC model was then analysed elastically and the integral of the absolute acceleration (i.e. cumulative absolute velocity [49]) was computed at the surface and compared against the linear frequency domain analyses to provide an adjustment factor that was used to scale the input motion for the linear, equivalent linear and prediction method, such that they had approximately the same input energy [50]. The mesh used for the FLAC analyses consisted of four $0.5 \mathrm{~m}$ elements wide, and the element heights in each layer were sized to be equal or less than $0.5 \mathrm{~m}$.

$\tau_{i n}=-k_{\tau} \cdot \rho \cdot V_{s} \cdot \dot{u}$

The linear and equivalent linear analyses were performed using the open-source python package, Pysra v0.3.0 [29]. For the linear analyses, all layers were reduced from their initial stiffness values and damping was set based on the peak ground acceleration (PGA). The shear modulus was reduced to $80 \%$ and damping of $3 \%$ for PGA less than $0.1 \mathrm{~g}$, for PGA less than $0.2 \mathrm{~g}$ the shear modulus was reduced to $50 \%$ and damping of $6 \%$, for larger PGA the shear modulus was reduced to $36 \%$ and damping of $10 \%$, consistent with Section 4.2 .3 of Eurocode Part 5 [51]. For the equivalent linear analyses the clay layers were modelled with the Modified Hyperbolic Soil Type using the expressions from Vardanega and Bolton [42] and a minimum damping of $2 \%$. The sand layer was modelled using the Modified Hyperbolic Soil Type model, where the curvature factor was set to 1.0 and the $\gamma_{\text {ref }}$ was set to best match the backbone response of numerical element tests of the soil at the centre of the layer performed in FLAC with the PM4Sand model. The damping and shear modulus reduction were computed using $65 \%$ of the maximum strain, and iterated to an error of less than $1 \%$ in the properties or 15 iterations.

Each of the 500 profiles was evaluated against one of 49 ground motions selected from the NGAWest ground motion database [32]. To select the ground motions the database was first filtered by the following criteria, and then one motion was selected as the closest to a set of 49 equally spaced peak ground acceleration (PGA) values between 0.1 and 0.49 , so that an even distribution of PGA values would be present in the database. No other criteria were used for the selection in an attempt to provide a wide, unbiased selection of ground motions. The two horizontal components were combined together to obtain the maximum rotated Arias Intensity [28] considering 100 potential angles.

The ground motion selection criteria:

1. Time-averaged shear-wave velocity of the $30 \mathrm{~m}$ directly below the recording station (Vs30) range: $180-400 \mathrm{~m} / \mathrm{s}$

2. Not a foreshock or aftershock event

3. From earthquake events with a magnitude larger than 5

4. Ground motion has a PGA higher than $0.10 \mathrm{~g}$

5. Have a lowest usable frequency less than or equal to $0.25 \mathrm{~Hz}$

6. Did not suffer from excessive disturbance during recording and contained the start of the shaking

The list of ground motions are listed in Table 2 along with the Vs30, the PGA, and the UKE (See Section 3, Equation (5)). The single degree- 
Table 2

Input ground motions for validation study.

\begin{tabular}{|c|c|c|c|c|c|c|c|c|c|}
\hline ID & Record & E. dist $[\mathrm{km}]$ & Mw & Vs30 $[\mathrm{m} / \mathrm{s}]$ & PGA [g] & UKE [m2/s2] & Earthquake & Year & Station \\
\hline 1 & 148 & 9.6 & 5.7 & 350 & 0.26 & 0.27 & Coyote Lake & 1979 & Gilroy A\#3 \\
\hline 2 & 159 & 2.6 & 6.5 & 242 & 0.32 & 0.69 & Imperial Valley-06 & 1979 & Agrarias \\
\hline 3 & 175 & 32.0 & 6.5 & 197 & 0.14 & 0.44 & Imperial Valley-06 & 1979 & El Centro A\#12 \\
\hline 4 & 240 & 2.8 & 5.7 & 382 & 0.55 & 0.20 & Mammoth Lakes-04 & 1980 & Convict Creek \\
\hline 5 & 313 & 19.9 & 6.6 & 361 & 0.35 & 0.46 & Corinth, Greece & 1981 & Corinth \\
\hline 6 & 449 & 43.5 & 6.2 & 289 & 0.14 & 0.08 & Morgan Hill & 1984 & Capitola \\
\hline 7 & 457 & 38.2 & 6.2 & 350 & 0.26 & 0.13 & Morgan Hill & 1984 & Gilroy A\#3 \\
\hline 8 & 461 & 3.9 & 6.2 & 282 & 0.32 & 0.42 & Morgan Hill & 1984 & Halls Valley \\
\hline 9 & 558 & 14.3 & 6.2 & 316 & 0.42 & 1.06 & Chalfant Valley-02 & 1986 & Zack Brothers Ranch \\
\hline 10 & 592 & 9.9 & 6.0 & 368 & 0.31 & 0.22 & Whittier Narrows-01 & 1987 & Arcadia - Campus Dr \\
\hline 11 & 626 & 21.3 & 6.0 & 301 & 0.40 & 0.21 & Whittier Narrows- 01 & 1987 & LA - 116th St School \\
\hline 12 & 692 & 11.7 & 6.0 & 339 & 0.43 & 0.46 & Whittier Narrows-01 & 1987 & Santa Fe Sp. E.Joslin \\
\hline 13 & 767 & 31.4 & 6.9 & 350 & 0.55 & 0.69 & Loma Prieta & 1989 & Gilroy A\#3 \\
\hline 14 & 770 & 39.9 & 6.9 & 334 & 0.32 & 0.28 & Loma Prieta & 1989 & Gilroy A\#7 \\
\hline 15 & 802 & 27.2 & 6.9 & 381 & 0.48 & 0.96 & Loma Prieta & 1989 & Saratoga - Aloha Ave \\
\hline 16 & 803 & 27.1 & 6.9 & 348 & 0.42 & 1.45 & Loma Prieta & 1989 & Saratoga - W Valley Coll. \\
\hline 17 & 838 & 94.8 & 7.3 & 370 & 0.14 & 0.35 & Landers & 1992 & Barstow \\
\hline 18 & 848 & 82.1 & 7.3 & 353 & 0.38 & 1.13 & Landers & 1992 & Coolwater \\
\hline 19 & 960 & 26.5 & 6.7 & 326 & 0.48 & 1.03 & Northridge-01 & 1994 & W Lost Canyon \\
\hline 20 & 1035 & 38.7 & 6.7 & 352 & 0.17 & 0.12 & Northridge- 01 & 1994 & Man. Beach - Man. \\
\hline 21 & 1082 & 12.3 & 6.7 & 321 & 0.37 & 0.85 & Northridge-01 & 1994 & Sun Valley - Roscoe Blvd \\
\hline 22 & 1115 & 42.1 & 6.9 & 256 & 0.15 & 0.52 & Kobe, Japan & 1995 & Sakai \\
\hline 23 & 1155 & 95.0 & 7.5 & 290 & 0.10 & 0.41 & Kocaeli, Turkey & 1999 & Bursa Tofas \\
\hline 24 & 1158 & 98.2 & 7.5 & 282 & 0.40 & 1.43 & Kocaeli, Turkey & 1999 & Duzce \\
\hline 25 & 1513 & 7.6 & 7.6 & 364 & 0.59 & 2.95 & Chi-Chi, Taiwan & 1999 & TCU079 \\
\hline 26 & 1605 & 1.6 & 7.1 & 282 & 0.48 & 2.84 & Duzce, Turkey & 1999 & Duzce \\
\hline 27 & 2007 & 54.6 & 5.3 & 196 & 0.14 & 0.02 & CA/Baja Border Area & 2002 & El Centro A\#11 \\
\hline 28 & 3636 & 68.2 & 6.3 & 315 & 0.19 & 0.32 & Taiwan SMART1(40) & 1986 & SMART1 I04 \\
\hline 29 & 3643 & 69.2 & 6.3 & 307 & 0.22 & 0.20 & Taiwan SMART1(40) & 1986 & SMART1 M02 \\
\hline 30 & 3653 & 70.0 & 6.3 & 285 & 0.20 & 0.19 & Taiwan SMART1(40) & 1986 & SMART1 O02 \\
\hline 31 & 4066 & 15.1 & 6.0 & 227 & 0.55 & 0.21 & Parkfield-02, CA & 2004 & Parkfield - FROELICH \\
\hline 32 & 4146 & 12.2 & 6.0 & 342 & 0.38 & 0.39 & Parkfield-02, CA & 2004 & Parkfield - UPSAR10 \\
\hline 33 & 4159 & 42.3 & 6.6 & 306 & 0.19 & 0.13 & Niigata, Japan & 2004 & FKS028 \\
\hline 34 & 4169 & 42.5 & 6.6 & 365 & 0.35 & 0.32 & Niigata, Japan & 2004 & FKSH21 \\
\hline 35 & 4210 & 13.6 & 6.6 & 332 & 0.64 & 1.29 & Niigata, Japan & 2004 & NIG020 \\
\hline 36 & 4212 & 30.1 & 6.6 & 193 & 0.33 & 0.28 & Niigata, Japan & 2004 & NIG022 \\
\hline 37 & 4866 & 8.5 & 6.8 & 338 & 0.35 & 0.76 & Chuetsu-oki & 2007 & Kawanishi Izumozaki \\
\hline 38 & 4889 & 58.1 & 6.8 & 315 & 0.37 & 0.37 & Chuetsu-oki & 2007 & Joetsu Otemachi \\
\hline 39 & 5263 & 22.6 & 6.8 & 274 & 0.26 & 0.46 & Chuetsu-oki & 2007 & NIG017 \\
\hline 40 & 5495 & 39.2 & 6.9 & 288 & 0.25 & 0.45 & Iwate & 2008 & AKTH19 \\
\hline 41 & 5616 & 88.6 & 6.9 & 364 & 0.20 & 0.09 & Iwate & 2008 & IWT007 \\
\hline 42 & 5664 & 32.1 & 6.9 & 361 & 0.43 & 5.13 & Iwate & 2008 & MYG005 \\
\hline 43 & 5669 & 75.2 & 6.9 & 275 & 0.11 & 0.17 & Iwate & 2008 & MYG010 \\
\hline 44 & 5814 & 51.2 & 6.9 & 248 & 0.34 & 2.21 & Iwate & 2008 & Furukawa Osaki City \\
\hline 45 & 5827 & 18.8 & 7.2 & 242 & 0.54 & 3.35 & El Mayor-Cucapah & 2010 & Michoacan de Ocampo \\
\hline 46 & 5829 & 32.4 & 7.2 & 242 & 0.41 & 2.67 & El Mayor-Cucapah & 2010 & RIITO \\
\hline 47 & 5836 & 55.3 & 7.2 & 265 & 0.45 & 1.46 & El Mayor-Cucapah & 2010 & Meloland Geot. Array \\
\hline 48 & 6927 & 33.8 & 7.0 & 263 & 0.42 & 2.23 & Darfield, New Zealand & 2010 & LINC \\
\hline 49 & 6962 & 26.9 & 7.0 & 296 & 0.45 & 3.03 & Darfield, New Zealand & 2010 & ROLC \\
\hline
\end{tabular}

of-freedom (SDOF) acceleration response spectra of the motions is shown in Fig. 10.

No deconvolution was performed as the characteristics of the site

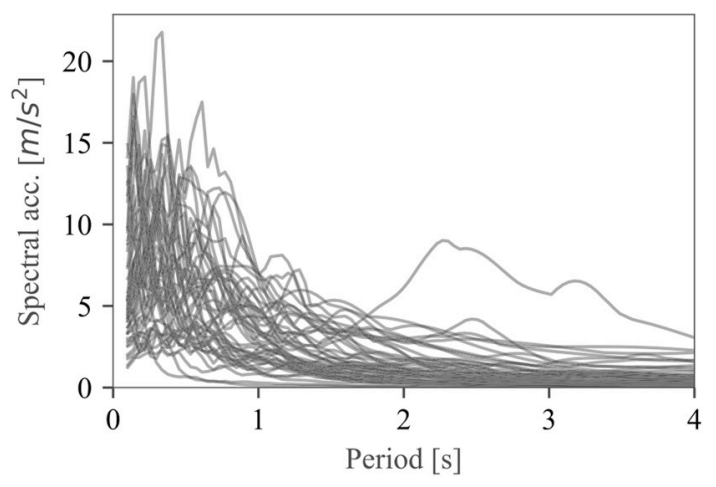

Fig. 10. SDOF acceleration response spectra of input motions used in validation study. where the recordings were taken were unknown, therefore the energy in these records at the depth of the base of the model may be slightly lower than expected for the same distance and magnitude of earthquake.

\subsection{Estimation of NCASE}

A comparison of the NCASE* throughout the depth of the profile obtained from equivalent linear, and total stress analyses as well as the NCASE estimated from the NSES method is shown for a single analysis in Fig. 11a) for a soil profile that had a $3 \mathrm{~m}$ clay layer of undrained strength of $32.5 \mathrm{kPa}$, initial shear modulus of $32.5 \mathrm{MPa}$ and dry unit weight of $15.8 \mathrm{kN} / \mathrm{m}^{3}$. The middle sand layer was $6 \mathrm{~m}$ thick and had a normalised shear modulus of 910 , relative density of $57 \%$ and saturated unit weight of $19.7 \mathrm{kN} / \mathrm{m}^{3}$. The base layer was a clay $17 \mathrm{~m}$ thick, and had an undrained strength of $183 \mathrm{kPa}$, initial shear modulus of $183 \mathrm{MPa}$ and saturated unit weight of $16.8 \mathrm{kN} / \mathrm{m}^{3}$. The ground motion was the Northridge-01 motion recorded in 1994 at Sun Valley - Roscoe Blvd station (ID1082). While all methods provide similar values of $N C A S E^{*}$ at all depths, the equivalent linear model slightly over predicts the influence of the change in stiffness across the boundary between the second 


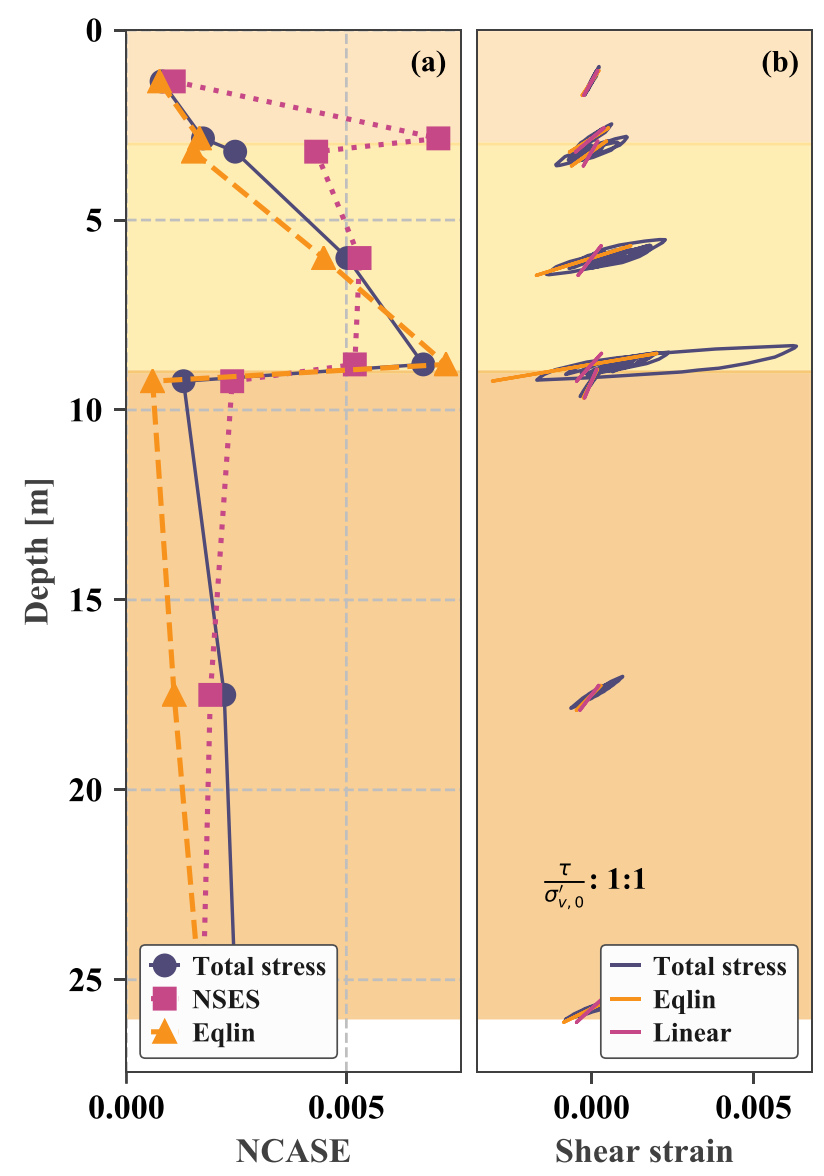

Fig. 11. Comparison of NCASE from equivalent linear analysis, total stress analysis and the NSES method in a single profile.

and third layers compared to the total stress analysis, since a large reduction in secant stiffness was observed for the middle layer for both the equivalent linear and total stress analyses (Fig. 11b). The overprediction in the change of strain energy across the layer boundary in the equivalent linear analysis is partly due to the stiffness reduction being applied for the whole ground motion, and therefore the amplification due to changes in stiffness would occur for even small amplitude waves. On the contrary the NSES method under-predicts the influence of this boundary since the elastic properties were used. The NSES method also over estimates the boundary between layers one and two, where the middle layer suffers more nonlinearity than the upper layer, thus reducing the stiffness contrast.

Fig. 12 shows a comparison of the NCASE* in the centre of the second layer from the total stress (TS), linear (linear) and equivalent linear (eqlin) analyses for all 500 soil profiles. In general the prediction of the NCASE* from the equivalent linear analysis and NSES method was close to the total stress analysis values, with the majority of values falling within the 1:2 and 2:1 bounds. Equivalent linear analysis has the drawback of amplifying the same deformation modes for the whole ground motion, and can over-damp high frequency content and under damp low frequency content. While the NSES method suffers from the outlined issues relating to stiffness contrast across layers and the level of energy dissipation. Remarkably the linear analysis provided reasonable estimates though under-predicted at large levels of strain energy. The mean normalised difference (absolute difference divided by the NCASE* from the total stress analysis) for all analyses was 0.31 for the NSES method compared to 0.30 and 0.40 for the equivalent linear and linear analyses respectively.

Fig. 13 shows the estimation of the strain energy in relation to the shear modulus reduction calculated at the centre of the second layer

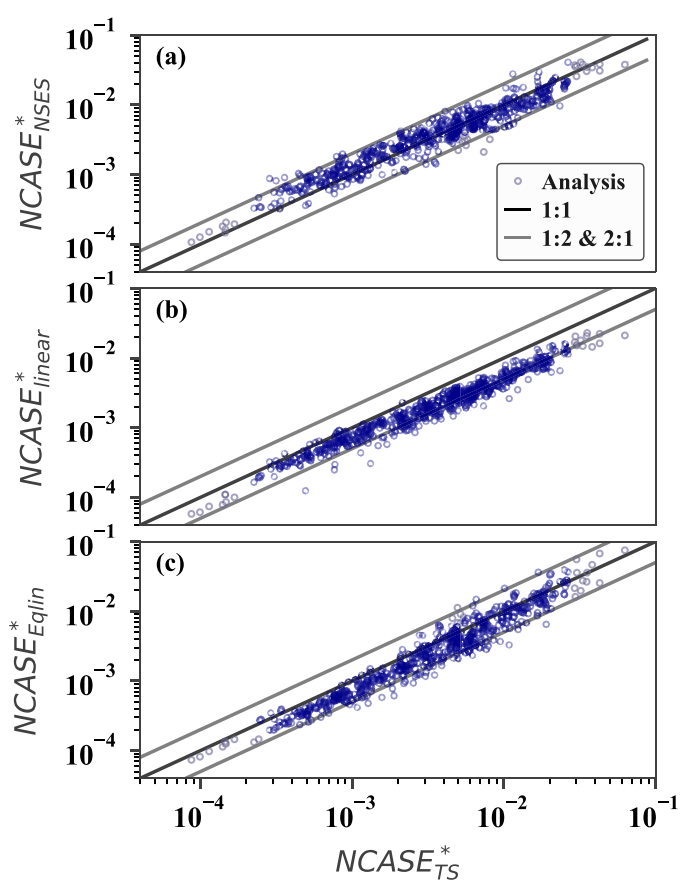

Fig. 12. Comparison of NCASE from total stress analyses with linear, equivalent and NSES methods.
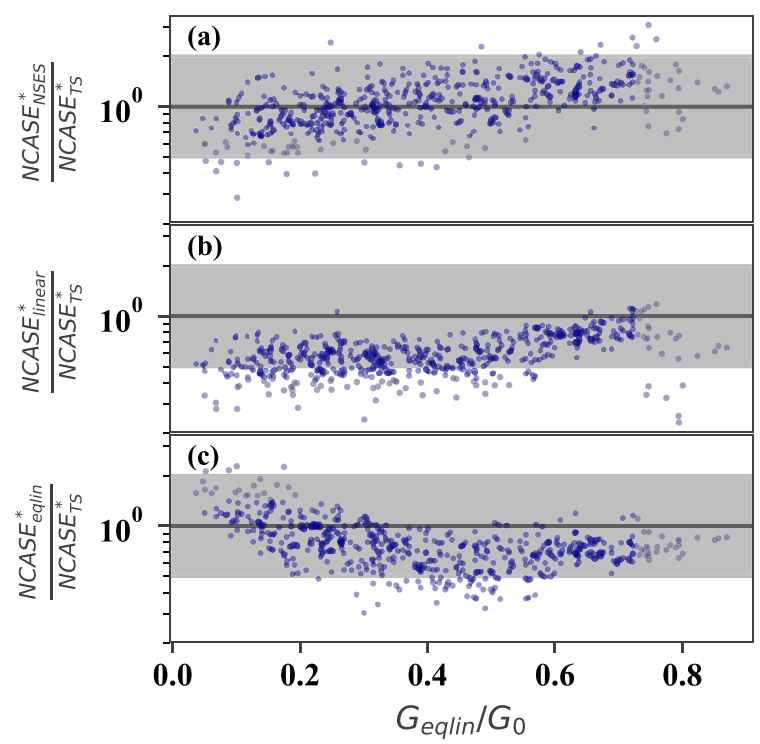

Fig. 13. Prediction of NCASE versus secant shear modulus reduction calculated from the equivalent linear analysis.

from the equivalent linear analysis. It can clearly be seen in the linear analyses and, to an extent, in the NSES method, that the prediction under-estimates when a large reduction was calculated in the equivalent linear analysis. The major contribution to this under-prediction is due to the reduction in apparent stiffness in the total stress analysis, which increases the strain energy (See Equation (12)). Fortunately this increase in energy due to stiffness reduction is counterbalanced by an increase in energy dissipation, leading to close estimates from the NSES method. For the equivalent linear analysis, the increase can be over amplified, since the stiffness remains constant for the whole time of the equivalent linear analysis.

Fig. 14 shows the $N C A S E^{*}$ in the centre of the second layer in the effective stress analyses (ES) up to the time of liquefaction or if no 


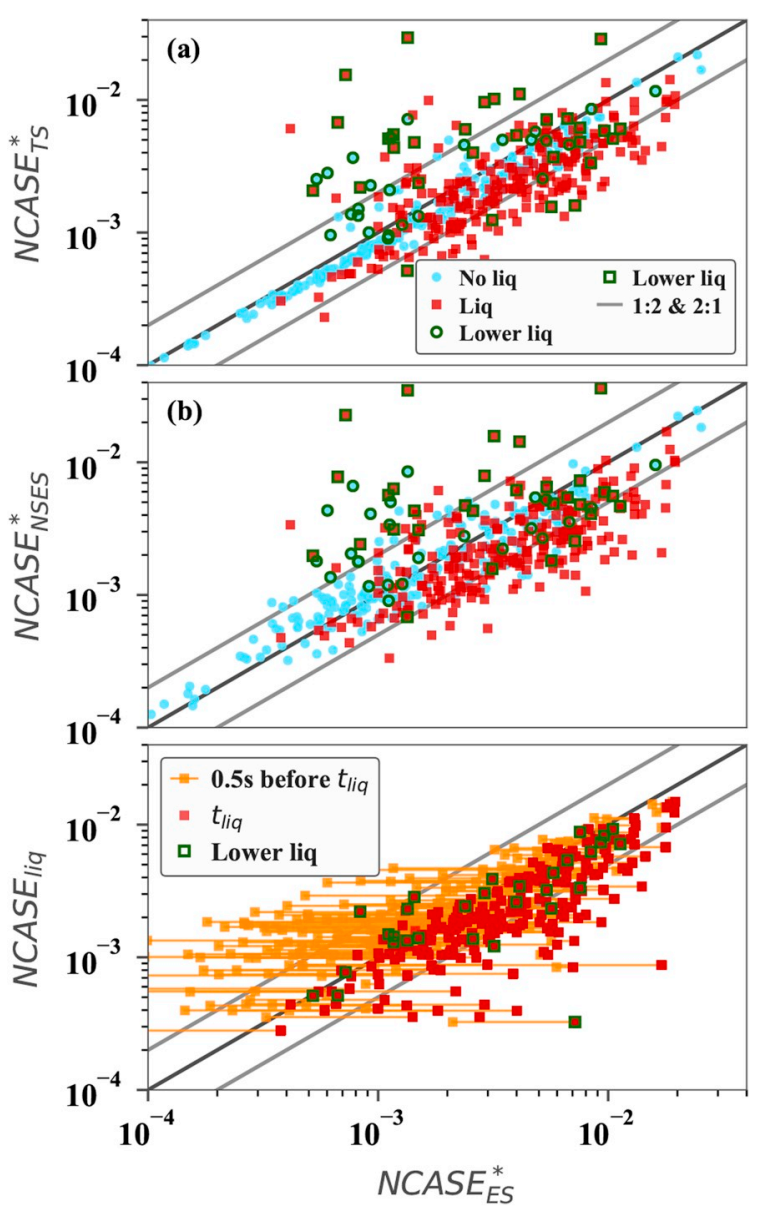

Fig. 14. Comparison of NCASE from effective stress analyses with NSES method, equivalent linear and element tests.

liquefaction happens, then the value at the end of the ground motion was used, compared to the NCASE* at the same time from the total stress analysis and the NSES method in subfigures $a$ and $b$ respectively. The time of liquefaction was taken as the first point where the mean effective stress dropped below $5 \mathrm{kPa}$, considered to be near the state of complete collapse of the simulated soil. In Fig. 14c, the NCASE* of all the liquefying analyses from the nonlinear effective stress analyses were compared to the $N C A S E_{\text {liq }}$ computed by running a series of numerical undrained direct simple shear element tests for the state of the soil at the centre of liquefiable layer at different cyclic stress ratios (0.04 to 0.6) and taking the mean $N C A S E_{\text {liq }}$ for all tests that liquefied between 2 and 40 cycles.

It can be seen that in the majority of simulations, the $N C A S E^{*}$ values in the effective stress and total stress analyses are very similar, highlighting that the NCASE* is largely unaffected by the build up of excess pore pressure due to the conservation of energy. Overall the effective stress analyses were slightly higher on average: for the motions that liquefy, the $N C A S E^{*}$ from the effective stress analyses were on average $66 \%$ greater than the complimentary total stress analysis, and for the noliquefaction simulations it was $18 \%$ greater. Interestingly, there were some motions that generated less NCASE* in the effective stress analysis for the same amount of time, in nearly all of these simulations liquefaction was triggered at the bottom of the liquefiable layer at least $2 \mathrm{~s}$ prior to the centre of the liquefiable layer ('Lower liq'). This suggests that some form of isolation has occurred limiting the seismic energy propagating into higher layers.

The NCASE* from the NSES method provides a reasonable estimate of the $N C A S E^{*}$ experienced in the effective stress analysis, but slightly more scatter compared to the total stress analyses. The mean normalised difference (absolute difference divided by the $N C A S E^{*}$ from the effective stress analysis) for all analyses was 0.65 for the NSES method compared to 0.56 from the total stress analyses. Again poor estimates are seen for soil profiles where liquefaction occurred in the base of the liquefiable layer at least $2 \mathrm{~s}$ prior to liquefaction at the centre. Although the scatter appears to be quite large, it is worth noting that to compare against corresponding stress based calculations, the square root of the error should be taken, due to the squared relationship between energy and stress. The scatter is also partly due to the rapid increase in NCASE* near liquefaction due to the partial collapse of the soil and/or a strong increase in seismic energy in the ground motion.

Fig. $14 \mathrm{c}$ shows the $N C A S E_{\text {liq }}$ from the elements tests compared to the $N C A S E^{*}$ from the effective stress analysis, with the right value being the actual $N C A S E^{*}$ at liquefaction, and the left value being the $N C A S E^{*}$ at $0.5 \mathrm{~s}$ prior to liquefaction. The large scatter in the results is clearly linked to a rapid change in $N C A S E^{*}$ in the effective stress analysis and results typically fall within a margin of $0.5 \mathrm{~s}$. This also reflects that the actual error in the prediction of the liquefying energy is actually a lot smaller than depicted in Fig. 14 a and b, and may be better represented by only the no-liquefaction simulations, where the mean normalised difference was 0.31 for the total stress analyses and 0.44 for the NSES method. It is interesting to note that liquefaction happened across a magnitude of $N C A S E^{*}$ from 0.0004 to 0.02 and that several of the 'Lower liq' analyses required less energy to liquefy in the one-dimensional analysis compared to the element tests (Fig. 14c), possibly due to upward pore water flow.

Fig. 15 shows the observed trends of the total stress analysis and NSES method versus the effective stress analyses for the analyses where liquefaction did not occur in the centre of the middle layer. Fig. 15a shows that the NCASE* from effective stress analysis was generally higher then total stress analysis when the pore pressure ratio $\left(r_{u}\right)$ exceeded 0.2 , this is due to the steep reduction in the shear modulus for even low values of excess pore pressure for the PM4Sand. Meanwhile the NSES method had larger NCASE* values at low $r_{u}$ values, where differences in the modelling of damping through viscous and Rayleigh damping models heavily influenced the estimated demand. Large differences can also be seen for soil profiles where liquefaction at the base occurred $2 \mathrm{~s}$ earlier, typically resulting in a reduction in $N C A S E^{*}$ in the effective stress analysis. The largest difference in the demand, for both the NSES and total stress values, was seen when the pore pressure ratio exceeded 0.7, due to the reduction in stiffness and increase in energy dissipation. While these effects increase the uncertainty in predictions, they could be rationally accounted for through estimates of stiffness reduction and energy dissipation as a function of the build-up of excess pore water pressure (e.g. Ref. [10]).

Fig. 15b shows a similar trend, where the NSES method overestimated the $N C A S E^{*}$ at low levels of nonlinearity (measured by the
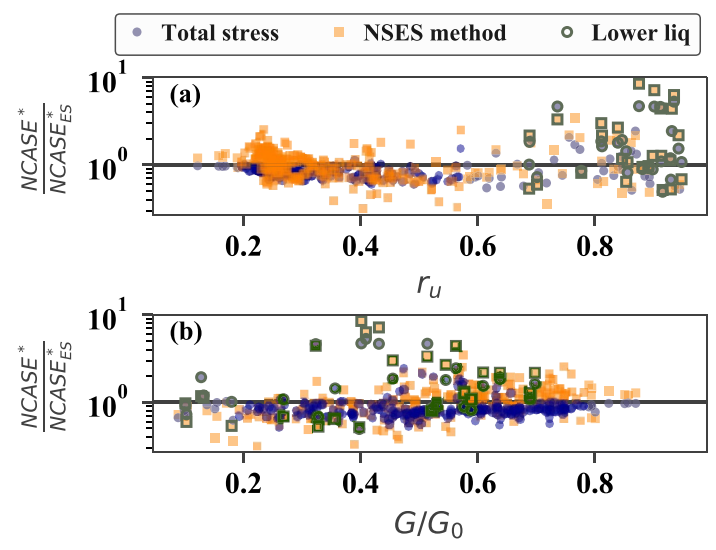

Fig. 15. Trends between the estimation of NCASE from total stress, NSES method and effective stress analyses. 
reduction in shear modulus from the equivalent linear analyses). As the nonlinearity increased there were no apparent differences between the total stress and NSES method and both slightly underestimated the demand. The equivalent linear stiffness reduction and pore pressure buildup are both correlated to stronger ground motions and weaker soil, and could be corrected for. However, given that the error is not large in the context of estimating pore pressure, and that there are still known biases between one-dimensional nonlinear analyses and field measurements from downhole arrays [52], these biases were not corrected for.

\subsection{Estimation of liquefaction triggering}

All the effective stress analysis results were used as a benchmark to determine whether the NSES method could accurately predict liquefaction in the middle layer (Fig. 16). The NSES method predicted 240 analyses to liquefy in the centre of the second layer, of which only 30 were incorrectly predicted, and of those, 16 reached $r_{u}$ greater than 0.8 , very close to liquefaction but not quite attaining the arbitrary $5 \mathrm{kPa}$ vertical effective stress threshold. From the 260 analyses that were predicted to not liquefy in the second layer, 43 did liquefy in the effective stress analysis, of which for 19 of them the calculated capacity was less than $25 \%$ higher than the predicted demand. It can also be noted from Fig. 16 that many of the mis-calculated results were strongly affected by liquefaction occurring at the bottom of the deposit ("Low liq").

\subsection{Estimation of excess pore pressure}

Given that soil softening can occur even at moderate levels of excess pore pressure build up, it is useful to be able to estimate the full time series of pore pressure build up. While the development of a robust relationship between excess pore pressure and $\frac{N C A S E^{*}}{N C A S E_{i q}}$ requires an extensive experimental campaign, for the purposes of validating the theoretical framework presented here, a simple square root relationship has been adopted (Equation (22)) and is shown in Fig. 17 to provide a reasonable estimate to the FLAC element test simulation results and the Coimbra Sand element tests results from Viana Da Fonseca et al. [22]. However, the Algeria Sand results showed a steeper initial increase in excess pore pressure, suggesting that the relationship is soil dependent.

$r_{u, i}=\min \left(\sqrt{\frac{N C A S E^{*}}{N C A S E_{l i q}}} \cdot r_{u, l i q}, 1.0\right)$

Using Equation (22) the excess pore pressure build-up could then be estimated throughout time and compared to the effective stress analyses. There were numerous trivial analyses where the NCASE* was significantly greater or less than $N C A S E_{\text {liq }}$, in which cases liquefaction triggered immediately or not at all. However, in Fig. 18 an example is presented where liquefaction was triggered late in the ground motion to

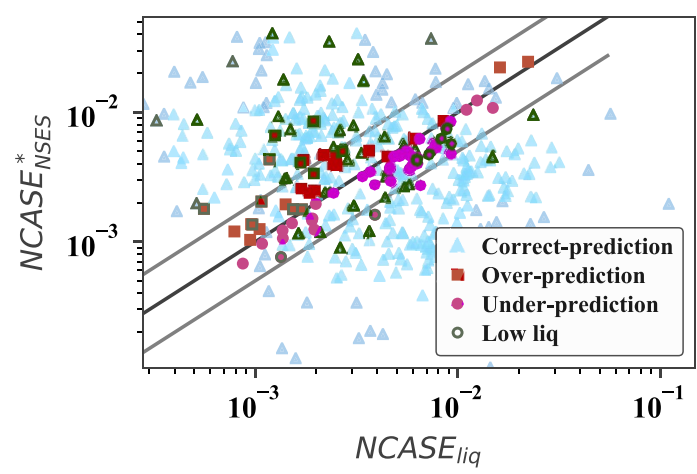

Fig. 16. Prediction of liquefaction at the centre of the liquefiable layer by the NSES method compared with effective stress analysis.

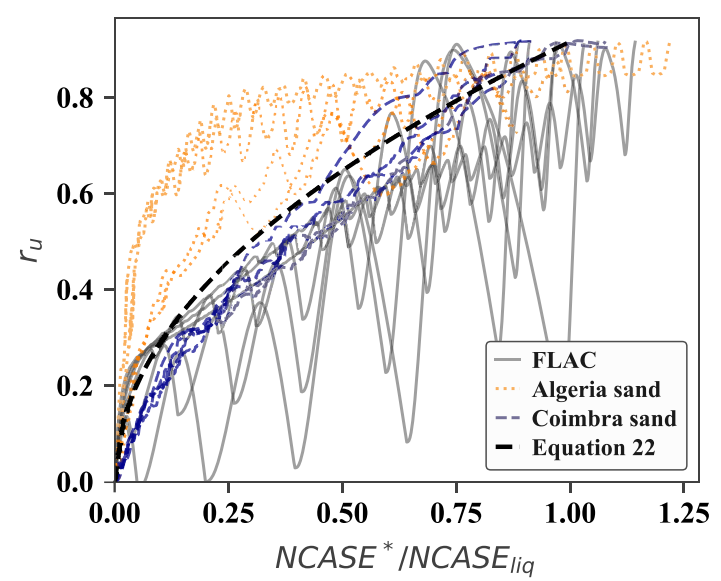

Fig. 17. Pore pressure ratio versus $\frac{N C A S E_{i}}{N C A S E_{i q}}$ for numerical element tests in FLAC (PM4Sand: $\mathrm{Dr}=0.7, G_{0}=1088, \mathrm{hpo}=0.55, \sigma^{\prime}{ }_{v, 0}=60 \mathrm{kPa}$ ), for CSS in Algeria sand $\left(\mathrm{Dr}=0.75, \sigma^{\prime}{ }_{v, 0}=100 \mathrm{kPa}\right)$, for CSS in Coimbra sand $\left(\mathrm{Dr}=0.45, \sigma^{\prime}{ }_{v, 0}=100 \mathrm{kPa}\right.$ ), and the square root relationship.

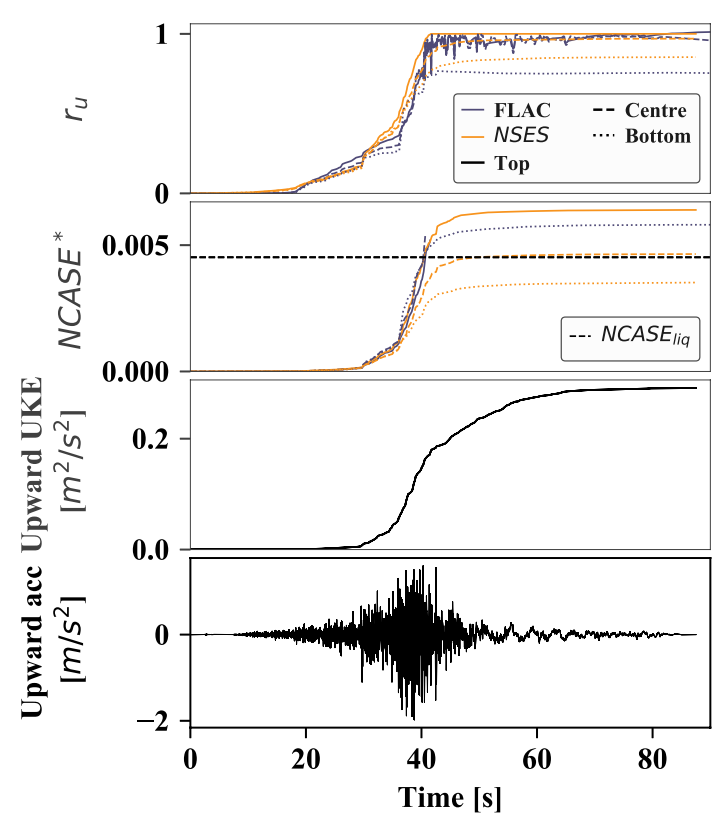

Fig. 18. Prediction of excess pore pressure for a single analysis.

demonstrate the accuracy of the proposed method. The soil profile used in Fig. 18 had a $5 \mathrm{~m}$ clay layer of undrained strength of $31.3 \mathrm{kPa}$, initial shear modulus of $32.5 \mathrm{MPa}$ and dry unit weight of $15.2 \mathrm{kN} / \mathrm{m}^{3}$. The middle sand layer was $8 \mathrm{~m}$ thick and had a normalised shear modulus of 1153 , relative density of $70 \%$ and saturated unit weight of $20.0 \mathrm{kN} / \mathrm{m}^{3}$. The base layer was a clay of $16 \mathrm{~m}$ and had an undrained strength of $186 \mathrm{kPa}$, initial shear modulus of $186 \mathrm{MPa}$ and saturated unit weight of $16.2 \mathrm{kN} / \mathrm{m}^{3}$. The ground motion was the El Mayor-Cucapah motion recorded in 2010 at Meloland Geot. Array station (ID5836). Both the prediction and the FLAC results show liquefaction occurring at approximately $40 \mathrm{~s}$. The bottom of the middle layer is accurately predicted to not liquefy, interestingly, the final estimate of ru is higher than the FLAC value but the estimation of strain energy is lower, possibly due to waterflow in the FLAC model. The demand and pore pressure experienced in the top and middle of the layer were almost identical in both the FLAC and NSES method. Overall the prediction was consistent with the behaviour observed in the FLAC analysis.

The prediction using the NSES method versus the effective stress results from all the analyses at the bottom, centre and top of the 
liquefiable layer are shown in Fig. 19 a and b, for the time of liquefaction and the maximum pore pressure ratio respectively. In general the time of liquefaction was well predicted for the bottom and middle of the layer, and slightly under predicted the time required to liquefy the soil in the top of the layer. This is most likely due to seismic isolation limiting the upward propagating energy from reaching the upper soil, as well as errors generated through changes in stiffness and dissipation of energy. There were also some soil profiles where the top of the layer liquefied in FLAC but was not predicted to liquefy, this was mostly due to upward pore water flow. The maximum pore pressure ratio showed consistent trends to those observed for the time to liquefaction, where a larger scatter and in general, an under-prediction of pore pressure was observed in the upper part of the layer, due to upward water flow. The role of pore water flow and seismic isolation were key parameters recognised by Cubrinovski et al. [53] that can strongly influence the triggering of liquefaction, and resulted in significant differences between the predicted performance using simple CPT-based triggering procedure [31] and observed ground damage during the Canterbury Earthquake Sequence (2010-2011). The accurate estimation of a build-up of energy and excess pore pressure throughout time and with depth using the NSES method could provide options for accounting for these system response effects in future research.

\section{Conclusion}

This paper presents a novel approach to estimate the build-up of excess pore water pressure and the time of liquefaction using kinetic energy and strain energy. The main findings of this work are:

1. The cumulative absolute change in strain energy required to liquefy the soil $\left(N C A S E_{\text {liq }}\right)$ was shown, through the investigation of existing element test data, to be nearly independent of the amplitude of loading and could be predicted from the dissipated energy required to liquefy the soil.

2. A unique equation was presented to quantify the cumulative absolute change in kinetic energy of a broadband travelling shear wave. The equation is also the direct measure of the total work done by the ground motion on a soil element.

3. The kinetic energy equation was used to estimate the strain energy throughout the depth of a soil deposit using the nodal surface energy spectrum, which accounted for the interaction of upward and downward propagating shear waves through the principle of conservation of energy. It was shown that the nodal surface energy spectrum provides an exact solution for the cumulative absolute strain and kinetic energy in a linear homogenous soil profile.

4. Simple corrections to the nodal surface energy spectrum procedure were developed to account for soil damping and changes in soil shear stiffness.

5. The developed procedure to predict the cumulative absolute strain energy was validated against results from linear, equivalent linear, nonlinear total stress and nonlinear effective stress one dimensional analyses for 500 randomly generated soil profiles with a variety of input ground motions. A high level of prediction was seen for the procedure even in the effective stress analyses where liquefaction occurred.

6. The estimated cumulative absolute strain energy was used to estimate the time of liquefaction of the effective stress analyses. In general, the prediction was consistent with the analyses, however, upward water flow and seismic isolation due to liquefaction occurring earlier lower in the deposit, were recognised as key parameters that influenced the prediction of liquefaction at a particular depth. The influence of stiffness contrasts between layers also requires further research to increase the robustness of predictions.

7. The build-up of excess pore pressure due to increasing cumulative absolute change in strain energy was approximated by a square root function, and combined with the proposed estimation of strain
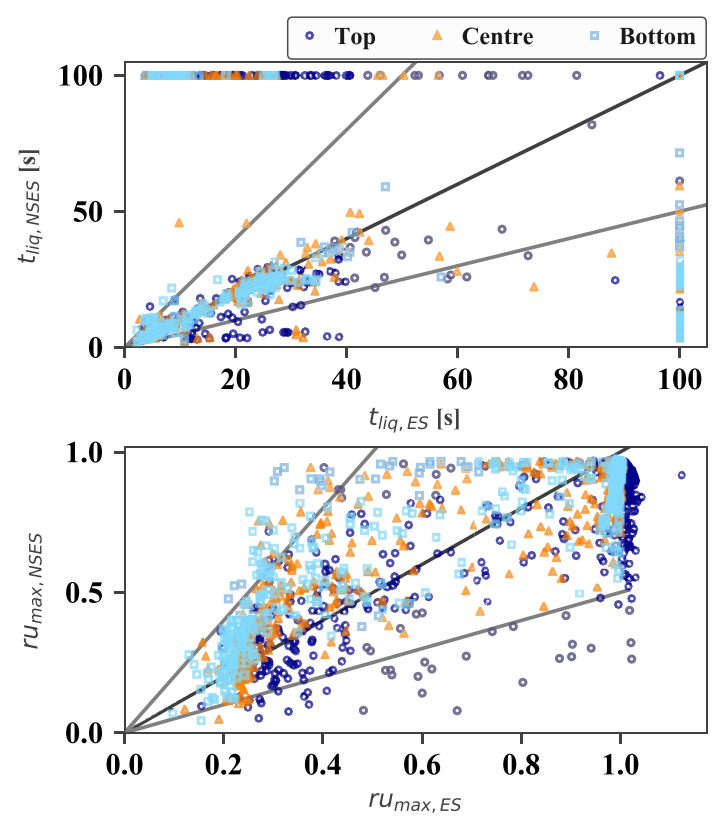

Fig. 19. Comparison of (a) time of liquefaction and (b) maximum excess pore pressure, from NSES method and effective stress analyses (ES).

energy. This approximation was shown to provide a reasonable prediction of the build up of excess pore pressure with respect to effective stress analyses.

8. The spectrum based solution provides an efficient, physically consistent and intuitive procedure for the assessment of the time of liquefaction, analogous to the single degree-of-freedom response spectrum used in structural design and assessment.

9. The NSES method for estimating the cumulative absolute strain energy has been implemented in the latest release of the python package liquepy.

\section{Acknowledgements}

This paper was produced as part of the LIQUEFACT project ("Assessment and mitigation of liquefaction potential across Europe: a holistic approach to protect structures/infrastructures for improved resilience to earthquake-induced liquefaction disasters") has received funding from the European Union's Horizon 2020 research and innovation programme under grant agreement No GAP-700748. This work was financially supported by: UID/ECI/04708/2019- CONSTRUCT Instituto de I\&D em Estruturas e Construções funded by national funds through the FCT/MCTES (PIDDAC). The experimental results were provided by the geotechnical lab in The University of Western Australia by Dr. Marisa Soares and due to a collaboration with Prof. Andy Fourie.

\section{References}

[1] Wotherspoon LM, Orense RP, Green RA, Bradley BA, Cox BR, Wood CM. Assessment of liquefaction evaluation procedures and severity index frameworks at Christchurch strong motion stations. Soil Dyn Earthq Eng 2015;79(Part B):335-46.

[2] Kramer SL, Hartvigsen AJ, Sideras SS, Ozener PT. Site response modeling in liquefiable soil deposits. In: 4th IASPEI/IAEE international; 2011.

[3] Jafarian Y, Mehrzad B, Lee CJ, Haddad AH. Centrifuge modeling of seismic foundation- soil-foundation interaction on liquefiable sand. Soil Dyn Earthq Eng 2017;97:184-204.

[4] Bird JF, Bommer JJ, Crowley H, Pinho R. Modelling liquefaction-induced building damage in earthquake loss estimation. Soil Dyn Earthq Eng 2006;26(1):15-30.

[5] Kramer SL, Sideras SS, Greenfield MW. The timing of liquefaction and its utility in liquefaction hazard evaluation. Soil Dyn Earthq Eng 2016;91(C):133-46.

[6] Bouckovalas GD, Tsiapas YZ, Zontanou VA, Kalogeraki CG. Equivalent linear computation of response spectra for liquefiable sites: the spectral envelope method. J Geotech Geoenviron Eng 2017;143(4). 04016115-12. 
[7] Lasley SJ, Green RA, Rodriguez-Marek A. Number of equivalent stress cycles for liquefaction evaluations in active tectonic and stable continental regimes. J Geotech Geoenviron Eng 2017;127(12):1-12.

[8] Green RA, Bommer JJ, Rodriguez-Marek A, Maurer BW, Stafford PJ, Edwards B, Kruiver PP, de Lange G, van Elk J. Addressing limitations in existing 'simplified' liquefaction triggering evaluation procedures: application to induced seismicity in the Groningen gas field. Bull Earthq Eng 2018:1-19.

[9] Seed HB. Representation of irregular stress time histories by equivalent uniform stress series in liquefaction analyses. 1975.

[10] Green RA, Terri GA. Number of equivalent cycles concept for liquefaction evaluations—revisited. J Geotech Geoenviron Eng 2005;131(4):477-88.

[11] Dobry R, Ladd RS, Yokel FY, Chung RM, Powell D. Prediction of pore water pressure buildup and liquefaction of sands during earthquakes by the Cyclic Strain Method. Gaithersburg, MD, Gaithersburg, MD: National Bureau of Standards; 1982. Technical report.

[12] Silver ML, Seed HB. Volume changes in sands during cyclic loading. 1971. trid.trb. org.

[13] Roscoe KH, Schofield AN, Geotechnique AT. Yielding of clays in states wetter than critical. Proc Inst Civ Eng Geotech Eng. 1963;13(3):211-40 (1963).

[14] Nemat-Nasser S, Shokooh A. A unified approach to densification and liquefaction of cohesionless sand in cyclic shearing. Can Geotech J 1979;16(4):659-78.

[15] Berrill JB, Davis RO. Energy dissipation and seismic liquefaction OF sands : revised model. Soils Found 1985;25(2):106-18.

[16] Towhata I, Ishihara K. Shear work and pore water pressure in undrained shear. Soils Found 1985;25(3):73-84.

[17] Green RA, Mitchell JK, Polito C. An energy-based excess pore pressure generation model for cohesionless soils. In: Balkema P, editor. Pro of the John Booker memorial symposium, sydney, Australia; 2000.

[18] Kokusho T. Liquefaction potential evaluations: energy-based method versus stressbased method. Can Geotech J 2013;50(10):1088-99.

[19] Azeiteiro RJN, Coelho PALF, Taborda DMG, Grazina JCD. Energy-based evaluation of liquefaction potential under non-uniform cyclic loading. Soil Dyn Earthq Eng 2017;92(C):650-65.

[20] Darendeli MB. Development of a new family of normalized modulus reduction and material damping curves. PhD thesis. The University of Texas at Austin; 2001.

[21] Kokusho T, Ito F, Nagao Y, Green AR. Influence of non/low-plastic fines and associated aging effects on liquefaction resistance. J Geotech Geoenviron Eng 2012;138(6):747-56.

[22] Viana Da Fonseca A, Soares M, Fourie AB. Cyclic DSS tests for the evaluation of stress densification effects in liquefaction assessment. Soil Dyn Earthq Eng 2015;75 (C):98-111.

[23] Timoshenko SP, Goodier JN. Theory of elasticity. 3 edition. McGraw-Hill Book Company; 1968.

[24] Jafarian Y, Vakili R, Abdollahi AS, Baziar MH. Simplified soil liquefaction assessment based on cumulative kinetic energy density: attenuation law and probabilistic analysis. Int J Geomech 2014;14(2):267-81.

[25] Kokusho T, Mimori Y. Liquefaction potential evaluations by energy-based method and stress-based method for various ground motions. Soil Dyn Earthq Eng 2015;75 (C):130-46.

[26] Sarma S. Energy flux of strong earthquakes. Phys Chem Earth 1971;11(3):159-73.

[27] Kayen RE, Mitchell JK. Assessment of liquefaction potential during earthquakes by Arias intensity. J Geotech Geoenviron Eng 1997;123(12):1162-74.

[28] Arias A. A measure of earthquake intensity. Seismic Des Nucl Power Plant 1970.

[29] Kottke A. Pysra v0.2.1. Pypi - Python package repository. 2018.

[30] Kokusho T. Liquefaction potential evaluations by energy-based method and stressbased method for various ground motions: supplement. Soil Dyn Earthq Eng 2017; 95(C):40-7.

[31] Boulanger RW, Idriss IM. CPT-based liquefaction triggering procedure. J Geotech Geoenviron Eng 2016;142(2). 04015065-11.

[32] Ancheta TD, Darragh R, Stewart JP, Emel S, Silva W, Chiou B, Wooddell KE, Graves RW, Kottke AR, Boore DM, Kishida T, Donahue J. PEER NGA-West2 database. USA: Technical Report 03; 2013.

[33] Kaklamanos J, Bradley BA, Thompson EM, Baise LG. Critical parameters affecting bias and variability in site-response analyses using KiK-net downhole array data. Bull Seismol Soc Am 2013;103(3):1733-49.

[34] Baker JW. Conditional mean spectrum: tool for ground-motion selection. J Struct Eng 2011;137(3):322-31.

[35] Boatwright J, Choy GL. Teleseismic estimates of the energy radiated by shallow earthquakes. J Geophys Res 1986;91(B2). 2095-18.

[36] Kramer SL, Mitchell RA. Ground motion intensity measures for liquefaction hazard evaluation. Earthq Spectra 2006;22(2):413-38.

[37] Dashti S, Karimi Z. Ground motion intensity measures to evaluate I: the liquefaction hazard in the vicinity of shallow-founded structures. Earthq Spectra 2017;33(1):241-76.

[38] Bullock Z, Dashti S, Liel A, Porter K, Karimi Z, Bradley BA. Ground-motion prediction equations for Arias intensity, cumulative absolute velocity, and peak incremental ground velocity for rock sites in different tectonic environments. Bull Seismol Soc Am 2017;107(5):2293-309.

[39] Boulanger RW, Ziotopoulou K. PM4SAND (version 3.1): a sand plasticity model for earthquake engineering applications. 2017. p. 1-113.

[40] ITASCA. FLAC2D - fast Lagrangian analysis of continua, version. 8.0. Minneapolis: Itasca. 2017.

[41] ITASCA. FLAC manual. 2017.

[42] Vardanega PJ, Bolton MD. Stiffness of clays and silts: normalizing shear modulus and shear strain. J Geotech Geoenviron Eng 2013;139(9):1575-89.
[43] Dafalias YF, Manzari M. Simple plasticity sand model accounting for fabric change effects. J Geotech Geoenviron Eng 2004.

[44] Kamai R, Boulanger RW. Simulations of a centrifuge test with lateral spreading and void redistribution effects. J Geotech Geoenviron Eng 2013;139(8):1250-61.

[45] Bray JD, Luque R. Seismic performance of a building affected by moderate liquefaction during the Christchurch earthquake. Soil Dyn Earthq Eng 2017;102: 99-111.

[46] Ziotopoulou K, Montgomery J. Numerical modeling of earthquake- induced liquefaction effects on shallow foundations. In: 16th world conference on earthquake engineering; 2017. p. 1-12. Santiago.

[47] Ziotopoulou K. Seismic response of liquefiable sloping ground: class A and C numerical predictions of centrifuge model responses. Soil Dyn Earthq Eng 2018; 113:744-57.

[48] Boulanger RW, Idriss IM. CPT AND SPT based liquefaction triggering procedures. 2014. Technical report.

[49] Reed JW, Kassawara RP. A criterion for determining exceedance of the operating basis earthquake. Nucl Eng Des 1990;123:387-96.

[50] Mejia LH, Dawson EM. Earthquake deconvolution for FLAC. In: 4th international symposium of numerical modelling in geomechanics, Minneapolis; 2006.

[51] EC 8 Part 5. Eurocode 8 Part 5: foundations, retaining structures and geotechnical aspects. European Standard; 2004. p. 1-68.

[52] Kaklamanos J, Bradley BA. Challenges in predicting seismic site response with 1D analyses: conclusions from 114 KiK-net vertical seismometer arrays. Bull Seismol Soc Am 2018;108(5A):2816-38.

[53] Cubrinovski M, Rhodes A, Ntritsos N, van Ballegooy S. System response of liquefiable deposits. Soil Dyn Earthq Eng 2018:1-18.

\section{GlossaryList of acronyms}

CRR: Cyclic resistance ratio

CSR: Cyclic stress ratio

CASE: Cumulative absolute change in strain energy

NCASE: Cumulative absolute strain energy normalised by the at rest vertical effective stress

$N_{\text {NASE }}$ liq NCASE required to cause liquefaction

$N C A S E^{*}$ : NCASE generated by a loading (e.g. ground motion)

$N C A S E_{T S}^{*}: N C A S E^{*}$ from total stress nonlinear analysis

$N C A S E_{\text {linear }}^{*}: N C A S E^{*}$ from linear analysis

$N C A S E_{E q l i n}^{*}: N C A S E^{*}$ from equivalent linear analysis

NCASE $_{N S E S}^{*}$ : NCASE* from NSES method

CAKE: Cumulative absolute change in kinetic energy

$U K E:$ Unit kinetic energy

NSES: Nodal surface energy spectrum

SDOF: Single degree-of-freedom

SPT: Standard penetration test

$P G A$ : Peak ground accelerationList of symbols

$M w$ : Earthquake moment magnitude

$D_{r}$ : Relative density

$d z$ : Small increment of distance

$E_{\text {diss }}:$ Dissipated energy

$f:$ Sine wave oscillation frequency

$k_{\tau}$ : Stress factor for inputting a motion along a compliant base

$G_{\text {layer, } .}$ : Shear modulus of the kth layer

$G_{\text {input }}$ : Shear modulus where NSES of the upward motion was computed

$g$ : Acceleration of gravity $\left(\approx 9.81 \mathrm{~m} / \mathrm{s}^{2}\right)$

$h_{p o}$ : Contraction rate parameter

$n_{\text {cycles,liq }}$ : Number of cycles to liquefaction

$N_{1,60, c s}$ : Clean sand equivalent normalised SPT blow count

$r_{u}$ : Excess pore pressure ratio, excess pore pressure divided by the initial vertical effective stress

$t_{\text {liq }}:$ Time of liquefaction triggering

$t_{h}$ : Travel time from the surface to the point of interest

$t_{H}$ : Travel time from the surface to the base of the soil profile

$\dot{u}_{i}$ : Upward ground motion horizontal velocity time series

$\ddot{u}_{i}$ : Upward ground motion horizontal acceleration time series

$V_{s}$ : Soil shear wave velocity

$I_{a}$ : Arias Intensity

ISV: Integral of the squared velocity

$C A V$ : Cumulative absolute velocity

$C A V_{5}$ : $C A V$ after application of $5 \mathrm{~cm} / \mathrm{s}^{2}$ threshold acceleration

$V_{S 30}$ : Time-averaged shear wave velocity of the $30 \mathrm{~m}$ soil deposit

$z$ : Depth of interest

$\eta_{t}$ : Damping reduction factor

$\eta_{\text {total }}:$ Ratio of the CAKE of the upward propagating shear wave at the surface compared to at the base of the profile

$\gamma$ : Shear strain

$\rho$ : Soil density

$\sigma^{\prime}, 0$ : At rest vertical effective stress

$\tau$ : Shear stress

$\xi$ : Viscous damping

$\lambda$ : Wavelength 